\title{
DYNAMIC OPTIMAL GRASPING OF A CIRCULAR OBJECT WITH GRAVITY USING ROBOTIC SOFT-FINGERTIPS
}

\author{
RODOLFO GARCÍA-RODRÍGUEZ ${ }^{a, *}$, VICTOR SEGOVIA-PALACIOS ${ }^{b}$, \\ VICENTE PARRA-VEGA ${ }^{b}$, MARCO VILLALVA-LUCIO $^{b}$ \\ ${ }^{a}$ Facultad de Ingeniería y Ciencias Aplicadas, Universidad de los Andes \\ Monseñor Álvaro del Portillo 12455, Las Condes, Santiago, Chile \\ e-mail: rgarcia1@miuandes.c1 \\ ${ }^{b}$ Robotics and Advanced Manufacturing, Research Center for Advanced Studies (Cinvestav) \\ Campus Saltillo, Av. Industria Metalurgica 1062, Ramos Arizpe, 25900, Mexico
}

\begin{abstract}
Object manipulation usually requires dexterity, encoded as the ability to roll, which is very difficult to achieve with robotic hands based on point contact models (subject to holonomic constraints). As an alternative for dexterous manipulation, deformable contact with hemispherical shape fingertips has been proposed to yield naturally a rolling constraint. It entails dexterity at the expense of dealing with normal and tangential forces, as well as more elaborated models and control schemes. Furthermore, the essential feature of the quality of grasp can be addressed with this type of robot hands, but it has been overlooked for deformable contact. In this paper, a passivity-based controller that considers an optimal grasping measure is proposed for robotic hands with hemispherical deformable fingertips, to manipulate circular dynamic objects. Optimal grasping that minimizes the contact wrenches is achieved through fingertip rolling until normal forces pass through the center of mass of the object, aligning the relative angle between these normal forces. The case of a circular object is developed in detail, though our proposal can be extended to objects with an arbitrary shape that admit a local decomposition by a circular curvature. Simulation and experimental results show convergence under various conditions, wherein rolling and tangent forces become instrumental to achieve such a quality of grasp.
\end{abstract}

Keywords: dynamic grasping, soft-fingertips, circular object.

\section{Introduction}

Most of the body of literature on dexterous hands assumes a frictionless rigid contact point between an object and fingers. It allows exerting a normal contact force (Cole et al., 1989), by using the classical DAE-21 formulation (Parra-Vega et al., 2001), wherein the tangential force is not modeled, but it is artificially considered a function of material properties at contact through the friction cone (Salisbury, 1982). In contrast, when rigid hemispherical fingertips are considered, tangent forces are introduced naturally based on the great deal of understanding of rolling contact mechanics (Jia, 2000; Harada and Kaneko, 2001; Ito et al., 2007; Nakashima et al., 2005). Interestingly, the dexterity of robotic hands increases when rolling constraints are taken into account (Marigo

\footnotetext{
* Corresponding author

${ }^{1}$ This signifies a system of index-2 differential algebraic equations.
}

and Bichi, 2007), because relocation of contact points can be carried out within a stable grasp regime, which is not possible with the point contact assumption. Such rolling motion was considered by Song et al. (2012) for force angle optimization, although for an object without gravity, in contrast to Ito et al. (2007), who include gravity by considering a-priori knowledge of stable contact points using a static analysis. Based on the concept of a virtual object, Stramigioli (2003) manipulates objects with arbitrary curvature (Wimboeck et al., 2006), without tangential forces by using a DAE-2 formulation, limiting its scope for dexterous grasping.

Notice that we humans grasp and manipulate everyday objects and tools of non-null curvature, which contribute to an ergonomic prehensile grasp that reduces the biomechanical stresses of hands and arms. Endowed by the hemispherical shape of our deformable fingertips, 
an essential feature of our hands, we exploit routinely object curvatures by fingertip rolling, without detaching (Akella and Cutkosky, 1989). To reproduce that in robotic hands, deformable fingertips have been studied recently to entail rolling motion (Ozawa et al., 2004; Nguyen et al., 2006), with independent control of normal and tangent forces (Arimoto et al., 2000). The resulting dynamic model is quite involved, just like its stability analysis, since it is subject to a holonomic constraint that models rolling at the velocity level. Surprisingly, the controller is not involved, showing the capability to grasp a planar dynamic object without gravity with a simple regulator that deals with environmental energy (Nguyen et al., 2006). This scheme stands for a more natural approach for dexterous manipulation because it exhibits the triplet rolling motion, tangent and normal forces (Arimoto et al., 2000). In this way, deformable fingertips provide a (simplistic) biomimetics-based performance (Arimoto, 2007); however, no measure of the quality of grasp has been addressed for dynamic objects subject to gravity.

Considering redundant robotic fingers with soft and hemispherical tips, in this paper we propose a 2D dexterous grasping controller of a dynamical circular object that is subject to the gravity effect, without any information about the object angle nor contact forces. The normal and tangential forces are controlled independently, such that the friction cone is shaped analytically without depending on an arbitrary static formulation (Cole et al., 1989), but within a dynamic setting. That is, our approach removes the zero curvature assumption of the object established in previous contributions, by including analytically the object dynamic equation for achieving a quality of grasp converging to a natural pose by rolling. The control design is based on the passivity approach to stabilize the object pose by regulating rolling and normal forces, similarly to Ozawa et al. (2004; 2005) or Nguyen et al. (2006); however, in contrast to them, an artificial potential energy is used to induce an optimal angle condition in the realm of quality of grasp based on an internal angle of the normal forces. It is shown that the desired tangential forces can be designed so as to compensate dynamically the external wrenches, including gravity, while simultaneously the normal forces are aligned collinear, passing through the center of mass $(C o M)$ of the object. In this way, an optimal grasp is achieved with a minimum contact wrench, where the angle condition stands for the index to qualify the grasping (Song et al., 2012; Kim et al., 2001).

With respect to the trajectory motion planning of this type of robotic hands, it is important to notice that our scheme only requires an initial stable grasp (contact and forces that assure grasp closure at initial conditions), as it is customary in robotic hands; however, the desired normal and tangential forces are automatically computed to stabilize the closed-loop system toward an optimal pose of the object that requires minimum normal forces. This stands for a principal characteristic of our approach, in contrast to complex trajectory motion planning of robotic hands based on contact points (Coelho and Grupen, 1994; Wen and Wu, 2012). Finally, simulations and experimental results are presented to highlight the viability of our approach, where the rolling motion and the tangential force become instrumental for dexterous tasks.

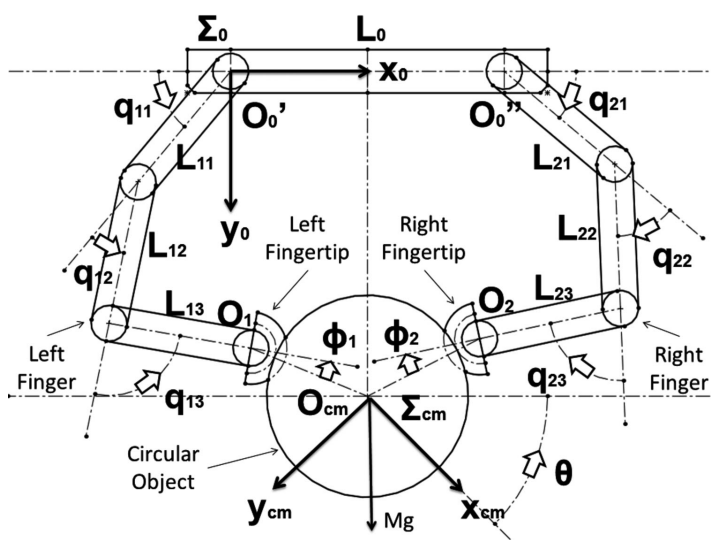

Fig. 1. Two robotic fingers grasping a circular object, where $\Sigma_{0}$ is a fixed/inertial coordinate frame.

\section{Constrained dynamical model}

2.1. Kinematic model. Consider a robotic hand with two planar fingers of three DoFs (degrees of freedom) each, whose end-effectors are covered with a hemispherical deformable homogeneous material. This system, the so-called hemispherical soft-fingertip robotic hand, is holding firmly a circular dynamic rigid object subject to the gravity effect; see Fig. 1] where $\boldsymbol{q}_{1}=$ $\left[q_{11}, q_{12}, q_{13}\right]^{T} \in \mathbb{R}^{3}$ and $\boldsymbol{q}_{2}=\left[q_{21}, q_{22}, q_{23}\right]^{T} \in \mathbb{R}^{3}$ are the generalized finger position coordinates for the right and left finger, respectively, $\theta \in \mathbb{R}$ stands for the object orientation/attitude, $\phi_{i} \in \mathbb{R}$ is the angle between the $i$-th normal force vector and the projected line of the last link of the $i$-th finger, and $\boldsymbol{p}=[x, y, \theta]^{T}=[\overline{\boldsymbol{p}}, \theta]^{T} \in$ $\mathbb{R}^{3}$ represents the generalized object position coordinates, with $\bar{p}=[x, y]^{T} \in \mathbb{R}^{2}$. Additionally, $L_{i j} \in \mathbb{R}$ stands for the $j$-th link length of the $i$-th finger, $L_{0} \in \mathbb{R}$ is the distance between the bases of each finger, $r \in \mathbb{R}$ and $R \in \mathbb{R}$ are the radii of the deformable fingertip and the object, respectively, $\boldsymbol{O}_{i}=\left[x_{i}, y_{i}\right]^{T} \in \mathbb{R}^{2}$ represents the center position of the deformable $i$-th fingertip, and $\boldsymbol{O}_{c m}=[x, y]^{T} \in \mathbb{R}^{2}$ is the center of mass of the object.

Notice that the $i$-th maximum fingertip radial deformation $\delta_{i}=\delta_{i}\left(\delta_{i}, \overline{\boldsymbol{p}}\right) \in \mathbb{R}$ can be defined as (see 


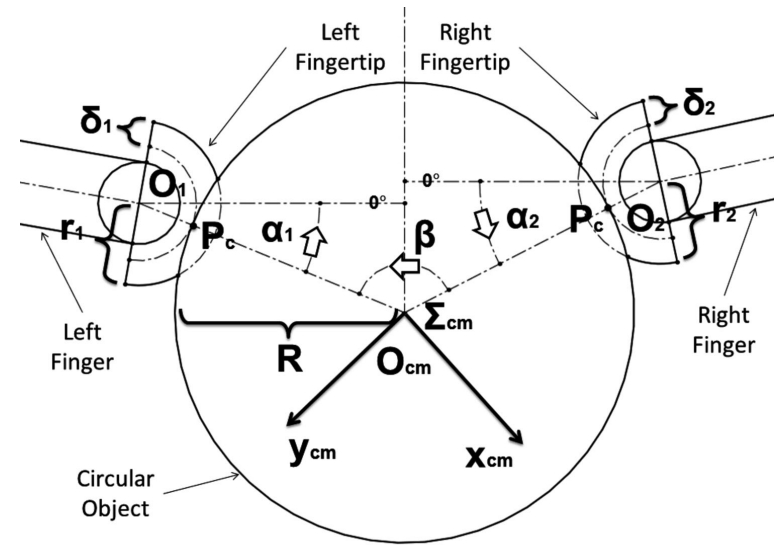

Fig. 2. Kinematic relationships at contact, where $\beta(\overline{\boldsymbol{\nu}})=\alpha_{1}-$ $\alpha_{2}+\pi \in \mathbb{R}$ represents the normal forces relative angle.

Fig. (2)

$$
\delta_{i}=\bar{R}+(-1)^{i}\left(\left[\begin{array}{cc}
\left(x-x_{i}\right) & \left(y-y_{i}\right)
\end{array}\right]\left[\begin{array}{c}
c\left(\alpha_{i}\right) \\
-s\left(\alpha_{i}\right)
\end{array}\right]\right),
$$

where $\bar{R}=r_{i}+R \in \mathbb{R}, s\left(\alpha_{i}\right)=\sin \left(\alpha_{i}\right) \in \mathbb{R}$, $c\left(\alpha_{i}\right)=\cos \left(\alpha_{i}\right) \in \mathbb{R}$ with $\alpha_{i}$ being the angle with respect to the horizontal of the $i$-th normal force, such that $\alpha_{i} \doteq \alpha_{i}\left(\boldsymbol{q}_{i}, \overline{\boldsymbol{p}}\right)=\tan ^{-1}\left(X_{i}\left(\boldsymbol{q}_{i}, \overline{\boldsymbol{p}}\right)\right) \in \mathbb{R}$, with

$$
X_{i}\left(\boldsymbol{q}_{i}, \overline{\boldsymbol{p}}\right)=-\left(\frac{y-y_{i}}{x-x_{i}}\right) \in \mathbb{R}
$$

(see Fig.22). In particular, the angle $\alpha_{i}$ will be instrumental in our approach to define the quality of grasp without a measurement of the object angle and arises as the fundamental difference from the other approaches (Ozawa et al., 2004; 2005; Nguyen et al., 2006).

Clearly, the hemispherical curvature of the deformable fingertip establishes that the angular rolling object velocity $\dot{\theta}$ can be related to $\dot{\phi}_{i}$ by $(-1)^{i} R \dot{\theta}=-r_{i} \dot{\phi}_{i}$, with respect to the inertial frame (Marigo and Bichi, 2007). This relation can be expressed by the following differentiable rolling constraint:

$$
\dot{\varphi}_{r_{i}}=(-1)^{i} R \frac{\mathrm{d}}{\mathrm{d} t} \theta+r_{i} \frac{\mathrm{d}}{\mathrm{d} t} \phi_{i}=0,
$$

where $\dot{\varphi}_{r_{i}}=\dot{\varphi}_{r_{i}}\left(\boldsymbol{q}_{i}, \boldsymbol{p}\right), \phi_{i}=\phi_{i}\left(\boldsymbol{q}_{i}, \overline{\boldsymbol{p}}\right)=\pi-$ $(-1)^{i} \alpha_{i}-\boldsymbol{q}_{i}^{T} \boldsymbol{e}_{i} \in \mathbb{R}$, and $\boldsymbol{e}_{i}=[1,1,1]^{T} \in \mathbb{R}^{3}$, for $i=1,2$. Furthermore, notice that (2) is integrable, whose vector-valued solution in fact stands for a rolling position holonomic constraint $\varphi_{r_{i}} \in \mathbb{R}$ as follows:

$$
\varphi_{r_{i}}=(-1)^{i} R \theta+r_{i} \phi_{i}+C_{\varphi_{r_{i}}}=0,
$$

where $\varphi_{r_{i}}=\varphi_{r_{i}}\left(\boldsymbol{q}_{i}, \boldsymbol{p}\right)$, and $C_{\varphi_{r_{i}}} \in \mathbb{R}$ stands for the integration constant.
2.2. Dynamic model. The kinetic energy $K=$ $K(\boldsymbol{q}, \dot{\boldsymbol{\nu}}) \in \mathbb{R}$ of the system is given by

$$
K(\boldsymbol{q}, \dot{\boldsymbol{\nu}})=\sum_{i=1}^{2} K_{i}+K_{0},
$$

where $\boldsymbol{q}=\left[\boldsymbol{q}_{1}^{T}, \boldsymbol{q}_{2}^{T}\right]^{T} \in \mathbb{R}^{6}, \boldsymbol{\nu}=\left[\boldsymbol{q}^{T}, \boldsymbol{p}^{T}\right]^{T} \in \mathbb{R}^{9}$, and $\dot{\boldsymbol{q}}=\left[\dot{\boldsymbol{q}}_{1}^{T}, \dot{\boldsymbol{q}}_{2}^{T}\right]^{T} \in \mathbb{R}^{6}, \dot{\boldsymbol{\nu}}=\left[\dot{\boldsymbol{q}}^{T}, \dot{\boldsymbol{p}}^{T}\right]^{T} \in \mathbb{R}^{9}$ are the generalized positions and velocities coordinates of the whole system, respectively, $K_{i}=\frac{1}{2} \dot{\boldsymbol{q}}_{i}^{T} H_{i}\left(\boldsymbol{q}_{i}\right) \dot{\boldsymbol{q}}_{i} \in$ $\mathbb{R}$ stands for the kinetic energy of the rigid $i$-th robot manipulator with $H_{i}\left(\boldsymbol{q}_{i}\right) \in \mathbb{R}^{3 \times 3}$ being the inertial positive definite matrix, $K_{0}=\frac{1}{2} \dot{\boldsymbol{p}} H_{0} \dot{\boldsymbol{p}} \in \mathbb{R}$ represents the kinetic energy of the circular object, with $H_{0}=$ $\operatorname{diag}(m, m, I) \in \mathbb{R}^{3 \times 3}$ being the constant object inertial matrix, $m \in \mathbb{R}$ the mass of the object and $I \in \mathbb{R}$ the mass moment of inertia of the object.

When a normal force is applied onto the circular object, it is assumed that the maximum force occurs at the contact point $P_{c}$ located along the line connecting the fingertip center with the $C o M$ of the object; see Fig. 2. Due to the deformation $\delta_{i}$ at fingertips, an $i$-th normal force $f_{i} \in \mathbb{R}$ arises, which can be modeled taking into account the following soft-fingertip deformation model (Arimoto, 2007):

$$
f_{i}=f_{i}\left(\delta_{i}, \overline{\boldsymbol{p}}\right)=k \delta_{i}^{2},
$$

where $k=2 \pi E \in \mathbb{R}$ and $E \in \mathbb{R}$ are the fingertip stiffness and the Young modulus of the soft material, respectively. Notice that (5) contributes with an elastic potential energy $P_{E_{i}}\left(\delta_{i}\right)$ at each fingertip, which can be obtained through integration as follows:

$$
\begin{aligned}
{\left[P_{E_{i}}\left(\delta_{i}\right)\right]_{0}^{t} } & =\int_{0}^{t} f_{i}\left(\delta_{i}\right) \frac{\mathrm{d}}{\mathrm{d} t} \delta_{i} \mathrm{~d} t \\
& =P_{E_{i}}\left(\delta_{i}\right)-P_{E_{i} 0}
\end{aligned}
$$

where

$$
P_{E_{i}}\left(\delta_{i}\right)=\frac{1}{3} \pi k \delta_{i}^{3}
$$

and

$$
P_{E_{i} 0}=P_{E_{i}}\left(\delta_{i}\left(\boldsymbol{q}_{i}(0), \overline{\boldsymbol{p}}(0)\right)\right)=\frac{1}{3} \pi k \delta_{i}^{3}\left(\boldsymbol{q}_{i}(0), \overline{\boldsymbol{p}}(0)\right)
$$

is the initial elastic potential energy at the contact. Thus, the total potential energy $P(\overline{\boldsymbol{\nu}}) \in \mathbb{R}$ of the whole system is given as

$$
P(\overline{\boldsymbol{\nu}})=\sum_{i=1}^{2} P_{E_{i}}\left(\delta_{i}\right)+\sum_{i=1}^{2} P_{G_{i}}(q)+P_{G_{0}}(y),
$$

where $\overline{\boldsymbol{\nu}}=\left[\boldsymbol{q}^{T}, \overline{\boldsymbol{p}}^{T}\right]^{T} \in \mathbb{R}^{8} ; P_{G_{i}}(\boldsymbol{q}) \in \mathbb{R}$ and $P_{G_{0}}(y)=$ $-M g y \in \mathbb{R}$ are the generalized potential energies induced by the Earth's gravitational field onto the $i$-th finger and onto the object, respectively. 
Additionally, notice that a workless constrained energy $S_{t}$ arises from the constrained forces (since the hemispherical $i$-th finger is constrained by the object). It is defined as

$$
S_{t}=\sum_{i=1}^{2} \varphi_{r_{i}}\left(\boldsymbol{q}_{i}, \boldsymbol{p}\right) \lambda_{i}=\varphi_{r}^{T} \boldsymbol{\lambda},
$$

where $\varphi_{r}=\left[\varphi_{r_{1}}, \varphi_{r_{2}}\right]^{T}=0 \in \mathbb{R}^{2}$ and $\boldsymbol{\lambda}=\left[\lambda_{1}, \lambda_{2}\right]^{T} \in$ $\mathbb{R}^{2}$ is the Lagrange multiplier with $\lambda_{i} \in \mathbb{R}$ being the magnitude of the tangential force applied at the contact point $P_{c i}$ of the $i$-th finger. Applying the variational principle to the constrained Lagrangian $L_{r}(\boldsymbol{\nu}, \dot{\boldsymbol{\nu}})=$ $L+S_{t}$, where $L=K(\boldsymbol{q}, \dot{\boldsymbol{\nu}})-P(\overline{\boldsymbol{\nu}})$ represents the Lagrangian, we obtain

$$
\int_{t_{1}}^{t_{2}}\left[\delta L+\boldsymbol{\varphi}_{r}^{T} \boldsymbol{\lambda}+\boldsymbol{u}^{T} \delta \boldsymbol{\nu}\right] \mathrm{d} t=0 .
$$

Solving (9) yields, according to the Euler-Lagrange modeling formalism,

$$
\frac{\mathrm{d}}{\mathrm{d} t}\left[\frac{\partial}{\partial \dot{\boldsymbol{\nu}}} L\right]-\frac{\partial}{\partial \boldsymbol{\nu}} L-\frac{\partial}{\partial \boldsymbol{\nu}} \boldsymbol{\varphi}_{r}^{T} \boldsymbol{\lambda}=\boldsymbol{u},
$$

where $\boldsymbol{u}=\left[\boldsymbol{\tau}_{d_{1}}^{T}, \boldsymbol{\tau}_{d_{2}}^{T}, \boldsymbol{\tau}_{z}^{T}\right]^{T}=\left[\boldsymbol{\tau}_{d_{1}}^{T}, \boldsymbol{\tau}_{d_{2}}^{T}, \mathbf{0}^{T}\right]^{T} \in \mathbb{R}^{9}$ stands for the generalized torque control inputs. With these definitions, (9) becomes

- dynamics of the $i$-th finger:

$$
\begin{aligned}
\boldsymbol{H}_{i}\left(\boldsymbol{q}_{i}\right) \ddot{\boldsymbol{q}}_{i}+ & \boldsymbol{C}_{i}\left(\boldsymbol{q}_{i}, \dot{\boldsymbol{q}}_{i}\right) \dot{\boldsymbol{q}}_{i}+\boldsymbol{g}_{i}\left(\boldsymbol{q}_{i}\right) \\
& =\boldsymbol{\tau}_{i}+(-1)^{i} f_{i}\left(\delta_{i}\right) \boldsymbol{J}_{i}^{T}\left(\boldsymbol{q}_{i}\right)\left[\begin{array}{c}
c\left(\alpha_{i}\right) \\
-s\left(\alpha_{i}\right)
\end{array}\right] \\
& +r_{i}\left(\boldsymbol{J}_{i}^{T}\left(\boldsymbol{q}_{i}\right) D_{i}^{-1}\left[\begin{array}{l}
s\left(\alpha_{i}\right) \\
c\left(\alpha_{i}\right)
\end{array}\right]-\boldsymbol{e}_{i}\right) \lambda_{i},
\end{aligned}
$$

- dynamics of the underactuated object:

$$
\begin{gathered}
\boldsymbol{H}_{0} \ddot{\boldsymbol{p}}-\left[\begin{array}{c}
0 \\
M g \\
0
\end{array}\right]-\sum_{i=1}^{2}\left((-1)^{i} f_{i}\left(\delta_{i}\right)\left[\begin{array}{c}
-c\left(\alpha_{i}\right) \\
s\left(\alpha_{i}\right) \\
0
\end{array}\right]\right) \\
=\boldsymbol{\tau}_{p}+\sum_{i=1}^{2}\left((-1)^{i} R\left[\begin{array}{l}
0 \\
0 \\
1
\end{array}\right]-r_{i} D_{i}^{-1}\left[\begin{array}{c}
s\left(\alpha_{i}\right) \\
c\left(\alpha_{i}\right) \\
0
\end{array}\right]\right) \lambda_{i},
\end{gathered}
$$

where $\boldsymbol{C}_{i}\left(\boldsymbol{q}_{i}, \dot{\boldsymbol{q}}_{i}\right)$ and $\boldsymbol{g}_{i}\left(\boldsymbol{q}_{i}\right)$ are the Coriolis matrix and gravitational forces/torques vector of the $i$-th finger, respectively; also notice that

$$
\boldsymbol{\tau}_{p}=[0,0,0]^{T} \in \mathbb{R}^{3}
$$

because the object is underactuated. Assuming reasonably that

$$
-\frac{\pi}{2} \leq \alpha_{i}\left(\boldsymbol{q}_{i}, \overline{\boldsymbol{p}}\right) \leq \frac{\pi}{2}
$$

for circular objects, because $r<R$, we have that

$$
\begin{aligned}
& \cos \left(\alpha_{i}\right)=\frac{1}{\sqrt{X_{i}^{2}\left(\boldsymbol{q}_{i}, \overline{\boldsymbol{p}}\right)+1}}=-(-1)^{i}\left(\frac{x-x_{i}}{D_{i}}\right), \\
& \sin \left(\alpha_{i}\right)=\frac{X_{i}}{\sqrt{X_{i}^{2}\left(\boldsymbol{q}_{i}, \overline{\boldsymbol{p}}\right)+1}}=-(-1)^{i}\left(\frac{y_{i}-y}{D_{i}}\right),
\end{aligned}
$$

with $D_{i}=D_{i}\left(\boldsymbol{q}_{i}, \overline{\boldsymbol{p}}\right)=\left\|\overline{\boldsymbol{x}}_{i}\left(\boldsymbol{q}_{i}\right)-\overline{\boldsymbol{p}}\right\|_{2}>0$ representing the distance from the object CoM to the position of the base center of the $i$-th deformable fingertip, for $\overline{\boldsymbol{x}}_{i}=$ $\left[x_{i}, y_{i}\right]^{T} \in \mathbb{R}^{2}$.

2.3. Some structural properties. From (10) and 111 we can elucidate some structural properties useful to design the control and guide the stability analysis.

System of index-2 differential algebraic equations (DAE-2). Notice that the underactuated object dynamics couple the finger dynamics into a hyperedundant closed-kinematic chain in the sense that the dimension of the operational space is much lower than the generalized coordinates dimension. Then the system (10)-11 represents an underactuated constrained hyperredundant DAE system that satisfies the constraint (2), as well as its derivatives. Despite the deformation at the fingertip, this stands for a very stiff system whose numerical solution requires a high-end stiff integrator for a well-posed numerical solution (Baumgarte, 1971).

Primary manifold. The state space of robotic fingers grasping an object is constrained to satisfy all the time the rolling constraint (2), which gives rise to the so-called primary constrained manifold $\mathcal{M}_{\varphi_{r}}$ defined by, for $i=$ 1, 2 (Yoshida et al., 2007),

$$
\mathcal{M}_{\varphi_{r}}=\left\{(\boldsymbol{\nu}, \dot{\boldsymbol{\nu}}) \in \mathbb{R}^{18}: \varphi_{r_{i}}=0, \dot{\varphi}_{r_{i}}=0\right\},
$$

where $\operatorname{dim}\left(\mathcal{M}_{\varphi_{r}}\right)=14$ due to $\{\boldsymbol{q}, \boldsymbol{p}\} \in \mathbb{R}^{9}$ minus the two constraints (at the position level), establishing the manifold where the solution evolves for all $t$.

Passivity and dissipativity. Let the input-output pair $(\boldsymbol{u}, \boldsymbol{y})$ be used to compute the power $\left\langle u_{i}, y_{i}\right\rangle$. We get

$$
\boldsymbol{u}^{T} \boldsymbol{y}=\frac{\mathrm{d}}{\mathrm{d} t} \mathcal{H},
$$

where the storage function $\mathcal{H}=\mathcal{H}(\overline{\boldsymbol{\nu}}, \dot{\boldsymbol{\nu}})=K(\boldsymbol{q}, \dot{\boldsymbol{\nu}})+$ $P(\overline{\boldsymbol{\nu}}) \in \mathbb{R}$ stands for the open-loop energy function of the system for $\boldsymbol{y}=\dot{\boldsymbol{\nu}} \in \mathbb{R}^{9}$, and $\boldsymbol{u} \in \mathbb{R}^{9}$. Thus, the constraint forces along the variational of the constraint velocity vanish. This implies that the integral of (13) yields $\mathcal{H}\left(\overline{\boldsymbol{\nu}}\left(t_{f}\right), \dot{\boldsymbol{\nu}}\left(t_{f}\right)\right)=\mathcal{H}\left(\overline{\boldsymbol{\nu}}\left(t_{0}\right), \dot{\boldsymbol{\nu}}\left(t_{0}\right)\right)$, which represents a passive lossless system. It is easy to show that if an affine joint or contact friction were considered, a dissipative map is enforced, suggesting that the control design must consider damping injection for stabilization purposes. 
2.4. Control problem statement. Consider DAE-2 dual redundant underactuated robotic fingers equipped with deformable and hemispherical tips in contact with a rigid circular object subject to gravity, see Fig. 1. Assume that generalized positions $\bar{\nu}$, kinematics maps, radii of soft-fingertips, gravitational forces/torques vectors of fingers, and the mass of the object are all known, but the following is unknown: the deformation, the elastic force and the contact area. Then, the control problem statement is as follows: Design a smooth control input $\tau_{i}$ to guarantee stable grasping such that the final pose implies a given quality of grasp with a minimum force.

\section{Control design}

3.1. Controller. Let the control law be

$$
\begin{aligned}
\boldsymbol{\tau}_{i}= & -\boldsymbol{K}_{q_{i}} \dot{\boldsymbol{q}}_{i}+\boldsymbol{g}_{i}\left(\boldsymbol{q}_{i}\right)-(-1)^{i} \boldsymbol{J}_{i}^{T}\left(\boldsymbol{q}_{i}\right)\left[\begin{array}{c}
c\left(\alpha_{i}\right) \\
-s\left(\alpha_{i}\right)
\end{array}\right] F_{d_{i}} \\
& -r_{i}\left(\boldsymbol{J}_{i}^{T}\left(\boldsymbol{q}_{i}\right) D_{i}^{-1}\left[\begin{array}{c}
s\left(\alpha_{i}\right) \\
c\left(\alpha_{i}\right)
\end{array}\right]-\boldsymbol{e}_{i}\right) \lambda_{d_{i}} \\
& -r_{i} \boldsymbol{J}_{i}^{T}\left(\boldsymbol{q}_{i}\right) D_{i}^{-1}\left[\begin{array}{l}
s\left(\alpha_{i}\right) \\
c\left(\alpha_{i}\right)
\end{array}\right] F_{\beta},
\end{aligned}
$$

where $\boldsymbol{K}_{q_{i}} \in \mathbb{R}^{3 \times 3}$ is a diagonal symmetric positive definite matrix, with $F_{d_{i}} \in \mathbb{R}$, and $\lambda_{d_{i}} \in \mathbb{R}$ being defined as

$$
\begin{aligned}
F_{d_{i}} & =f_{d}\left(1+(-1)^{i} \frac{1}{2} \Delta C S_{i}\right)-(-1)^{i} \frac{1}{2} \operatorname{Mgs}\left(\alpha_{i}\right) \\
\lambda_{d_{i}} & =-F_{\beta}-f_{d} \frac{1}{2} \frac{D_{i}}{r_{i}} \Delta S C_{i}+\frac{1}{2} \frac{D_{i}}{r_{i}} M g c\left(\alpha_{i}\right)
\end{aligned}
$$

for $f_{d} \in \mathbb{R}^{+}$standing for a constant normal force that assures grasp closure (Shapiro, 2001), and

$$
\begin{aligned}
& \Delta C S_{i}=\Delta C_{\alpha} c\left(\alpha_{i}\right)+\Delta S_{\alpha} s\left(\alpha_{i}\right), \\
& \Delta S C_{i}=\Delta S_{\alpha} c\left(\alpha_{i}\right)-\Delta C_{\alpha} s\left(\alpha_{i}\right),
\end{aligned}
$$

with $\Delta C_{\alpha}=c\left(\alpha_{1}\right)-c\left(\alpha_{2}\right) \in \mathbb{R}$ and $\Delta S_{\alpha}=s\left(\alpha_{1}\right)-$ $s\left(\alpha_{2}\right) \in \mathbb{R}$. Finally, the variable $F_{\beta} \in \mathbb{R}$ is introduced to qualify grasp, whose effect is to modulate tangential forces depending on the relative angle between normal forces. In this way, $F_{\beta}$ is defined as follows:

$$
F_{\beta}=K_{\beta_{p}} \Delta \bar{\beta}+K_{\beta_{v}}\left(r_{1} \dot{\boldsymbol{q}}_{1}^{T} \boldsymbol{e}_{1}+r_{2} \dot{\boldsymbol{q}}_{2}^{T} \boldsymbol{e}_{2}\right)
$$

where $K_{\beta_{p}} \in \mathbb{R}^{+}, K_{\beta_{v}} \in \mathbb{R}^{+}$, and $\Delta \bar{\beta}=\bar{\beta}-\beta_{d}$ stands for the angle error for stable grasping with

$$
\bar{\beta}=r_{1} \alpha_{1}-r_{2} \alpha_{2}+\pi
$$

modeling the internal angle between normal force vectors weighted by fingertip radii; see Fig. 2. The variable $\bar{\beta}$ can be interpreted as a grasping quality index in terms of internal squeezing forces as a rotational spring pivoting at the object CoM (Song et al., 2012). Clearly, an optimal stable grasp arises for $\beta_{d}=\pi$ wherein normal forces point toward the $\operatorname{CoM}$, thus representing the pose that requires the minimum forces to grasp an object. Notice that $\beta_{d}$ can be set to another value according to given criteria and relationship between fingertip radii.

Remark 1. Notice that, when $\Delta \bar{\beta}$ and $\dot{\nu}$ converge to zero, $\lambda_{d_{i}}$ and $F_{d_{i}}$ converge to constant values, exactly with a pose corresponding to collinear normal forces pointing toward the $C o M$, which for the circular object stands for an optimal grasp. Also, note that gravity compensation of the object is carried out through both $F_{d_{i}}$ and $\lambda_{d_{i}}$. Finally, external desired $f_{d}$ and $\beta_{d}$ are constant, meaning that the closed-loop system becomes autonomous at equilibrium. Interestingly enough, desired tangent forces are intuitively directly depending on the object angle error $\Delta \bar{\beta}$; the larger the error, the larger $\lambda_{d_{i}}$ becomes, and vice-versa.

3.2. Closed-loop system. Substituting (14) into (10), we obtain the following closed-loop dynamics:

- closed-loop fingers dynamics,

$$
\begin{gathered}
\boldsymbol{H}_{i}\left(\boldsymbol{q}_{i}\right) \ddot{\boldsymbol{q}}_{i}+\boldsymbol{C}_{i}\left(\boldsymbol{q}_{i}, \dot{\boldsymbol{q}}_{i}\right) \dot{\boldsymbol{q}}_{i}-(-1)^{i} \Delta f_{i} \boldsymbol{J}_{i}^{T}\left(\boldsymbol{q}_{i}\right)\left[\begin{array}{c}
c\left(\alpha_{i}\right) \\
-s\left(\alpha_{i}\right)
\end{array}\right] \\
+\boldsymbol{K}_{q_{i}} \dot{\boldsymbol{q}}_{i}-r_{i}\left(\boldsymbol{J}_{i}^{T}\left(\boldsymbol{q}_{i}\right) D_{i}^{-1}\left[\begin{array}{l}
s\left(\alpha_{i}\right) \\
c\left(\alpha_{i}\right)
\end{array}\right]-\boldsymbol{e}_{i}\right) \Delta \lambda_{i} \\
+r_{i} \boldsymbol{J}_{i}^{T}\left(\boldsymbol{q}_{i}\right) D_{i}^{-1}\left[\begin{array}{l}
s\left(\alpha_{i}\right) \\
c\left(\alpha_{i}\right)
\end{array}\right] F_{\beta}=\mathbf{0},
\end{gathered}
$$

- closed-loop underactuated object dynamics,

$$
\begin{gathered}
M \ddot{x}-\Delta f_{1} c\left(\alpha_{1}\right)+\Delta f_{2} c\left(\alpha_{2}\right)+\bar{r}_{1} s\left(\alpha_{1}\right) \Delta \lambda_{1} \\
+\bar{r}_{2} s\left(\alpha_{2}\right) \Delta \lambda_{2}-\bar{r}_{1} s\left(\alpha_{1}\right)+\bar{r}_{2} s\left(\alpha_{2}\right) F_{\beta}=0, \\
M \ddot{y}+\Delta f_{1} s\left(\alpha_{1}\right)-\Delta f_{2} s\left(\alpha_{2}\right)+\bar{r}_{1} c\left(\alpha_{1}\right) \Delta \lambda_{1} \\
+\bar{r}_{2} c\left(\alpha_{2}\right) \Delta \lambda_{2}-\bar{r}_{1} c\left(\alpha_{1}\right)+\bar{r}_{2} c\left(\alpha_{2}\right) F_{\beta}=0, \\
I \ddot{\theta}+R\left(\Delta \lambda_{1}-\Delta \lambda_{2}\right)+R f_{d} \frac{1}{2} D_{12} C S \\
+R \frac{1}{2} M g D C_{12}=0,
\end{gathered}
$$

where $\Delta f_{i}=f_{i}\left(\delta_{i}\right)-F_{d_{i}} \in \mathbb{R}$ is the normal force error, and $\Delta \lambda_{i}=\lambda_{i}-\lambda_{d_{i}} \in \mathbb{R}$ stands for the tangential force error, and (15)-(18) have been used to obtain (22)-(24). In the latter, $\bar{r}_{i}=r_{i} D_{i}^{-1} \in \mathbb{R}, D_{12}=\bar{r}_{1}^{-1}\left(\boldsymbol{q}_{1}, \overline{\boldsymbol{p}}\right)-$ $\bar{r}_{2}^{-1}\left(\boldsymbol{q}_{2}, \overline{\boldsymbol{p}}\right) \in \mathbb{R}, C S=c\left(\alpha_{1}\right) s\left(\alpha_{2}\right)-c\left(\alpha_{2}\right) s\left(\alpha_{1}\right) \in \mathbb{R}$ and $D C_{12}=\bar{r}_{1}^{-1}\left(\boldsymbol{q}_{1}, \overline{\boldsymbol{p}}\right) c\left(\alpha_{1}\right)-\bar{r}_{2}^{-1}\left(\boldsymbol{q}_{2}, \overline{\boldsymbol{p}}\right) c\left(\alpha_{2}\right) \in \mathbb{R}$. Finally, 21) (24) can be expressed in compact form to stand for the complete closed-loop error dynamics as follows:

$$
\boldsymbol{H} \ddot{\boldsymbol{\nu}}+\boldsymbol{C} \dot{\boldsymbol{\nu}}+\boldsymbol{K} \dot{\boldsymbol{\nu}}+\boldsymbol{A} \Delta \Lambda=\boldsymbol{u}_{c l},
$$


where $\boldsymbol{u}_{c l} \equiv 0$ is useful for stability analysis purposes only, $\ddot{\boldsymbol{q}}=\left[\ddot{\boldsymbol{q}}_{1}^{T}, \ddot{\boldsymbol{q}}_{2}^{T}\right]^{T} \in \mathbb{R}^{6}, \ddot{\boldsymbol{\nu}}=\left[\ddot{\boldsymbol{q}}^{T}, \ddot{\boldsymbol{p}}^{T}\right]^{T} \in \mathbb{R}^{9}$ are the generalized accelerations of the system,

$$
\begin{gathered}
\boldsymbol{H}=\operatorname{diag}\left(\boldsymbol{H}_{1}\left(\boldsymbol{q}_{1}\right), \boldsymbol{H}_{2}\left(\boldsymbol{q}_{2}\right), \boldsymbol{H}_{0}\right) \in \mathbb{R}^{9 \times 9}, \\
\boldsymbol{C}=\operatorname{diag}\left(\boldsymbol{C}_{1}\left(\boldsymbol{q}_{1}, \dot{\boldsymbol{q}}_{1}\right), \boldsymbol{C}_{2}\left(\boldsymbol{q}_{2}, \dot{\boldsymbol{q}}_{2}\right), \mathbf{0}_{3 \times 3}\right) \in \mathbb{R}^{9 \times 9},
\end{gathered}
$$

and

$$
\boldsymbol{K}=\operatorname{diag}\left(\boldsymbol{K}_{q_{1}}, \boldsymbol{K}_{q_{2}}, \mathbf{0}_{3 \times 3}\right) \in \mathbb{R}^{9 \times 9} .
$$

The coupling matrix $\boldsymbol{A}=\operatorname{diag}\left(\boldsymbol{A}_{1}, \boldsymbol{A}_{2}\right) \in \mathbb{R}^{9 \times 7}$ is given by

$$
\begin{aligned}
& \boldsymbol{A}_{1} \\
& =\left[\begin{array}{ccc}
\boldsymbol{J}_{1}^{T}\left[\begin{array}{c}
\cos \left(\alpha_{1}\right) \\
-\sin \left(\alpha_{1}\right)
\end{array}\right] & \mathbf{0}_{n \times 1} & -\frac{\partial}{\partial \boldsymbol{q}_{1}} \varphi_{r_{1}} \\
\mathbf{0}_{n \times 1} & \boldsymbol{J}_{2}^{T}\left[\begin{array}{c}
-\cos \left(\alpha_{2}\right) \\
\sin \left(\alpha_{2}\right)
\end{array}\right] & \mathbf{0}_{n \times 1} \\
-\cos \left(\alpha_{1}\right) & \cos \left(\alpha_{2}\right) & -\frac{\partial}{\partial \boldsymbol{x}} \varphi_{r_{1}} \\
\sin \left(\alpha_{1}\right) & -\sin \left(\alpha_{2}\right) & -\frac{\partial}{\partial \boldsymbol{y}} \varphi_{r_{1}} \\
0 & 0 & R
\end{array}\right],
\end{aligned}
$$

$\boldsymbol{A}_{2}=$

$$
\left[\begin{array}{cccc}
\mathbf{0}_{n \times 1} & -\boldsymbol{J}_{1}^{T} \frac{\partial}{\partial \boldsymbol{z}} \varphi_{r_{1}} & \mathbf{0}_{n \times 1} & \mathbf{0}_{n \times 1} \\
-\frac{\partial}{\partial \boldsymbol{q}_{2}} \varphi_{r_{2}} & -\boldsymbol{J}_{2}^{T} \frac{\partial}{\partial \boldsymbol{z}} \varphi_{r_{2}} & \mathbf{0}_{n \times 1} & \mathbf{0}_{n \times 1} \\
-\frac{\partial}{\partial \boldsymbol{x}} \varphi_{r_{2}} & \frac{\partial}{\partial \boldsymbol{x}} \varphi_{r_{1}}+\frac{\partial}{\partial \boldsymbol{x}} \varphi_{r_{2}} & 0 & 0 \\
-\frac{\partial}{\partial \boldsymbol{y}} \varphi_{r_{2}} & \frac{\partial}{\partial \boldsymbol{y}} \varphi_{r_{1}}+\frac{\partial}{\partial \boldsymbol{y}} \varphi_{r_{2}} & 0 & 0 \\
-R & 0 & R f_{d} \frac{1}{2} C S & R \frac{1}{2} M g
\end{array}\right]
$$

where the arguments of functions are omitted for convenience, and the vector $\Delta \Lambda \in \mathbb{R}^{7}$ stands for the seven control objectives, given by

$$
\Delta \Lambda=\left[\begin{array}{lllllll}
\Delta f_{1} & \Delta f_{2} & \Delta \lambda_{1} & \Delta \lambda_{2} & F_{\beta} & D_{12} & D C_{12}
\end{array}\right]^{T} .
$$

Now, we are ready to state the main result.

\subsection{Main result}

Theorem 1. Let

$$
\Omega_{0}=\left\{\boldsymbol{\nu}, \dot{\boldsymbol{\nu}}: \delta_{i}=\sqrt{\frac{f_{i}}{k}} \geq 0,\{\boldsymbol{\nu}, \dot{\boldsymbol{\nu}}\} \in \mathcal{M}_{\varphi_{r}}\right\}
$$

be a non-empty connected set. Consider the closed-loop error dynamics. Then, if initial conditions $\boldsymbol{\nu}(0) \in \Omega_{0}$ are small, the following basic constrained manifold:

$$
\begin{aligned}
\mathcal{M}_{c}=\left\{\boldsymbol{\nu}, \dot{\boldsymbol{\nu}}: \varphi_{r_{i}}=0, \dot{\varphi}_{r_{i}}=0\right. \\
\\
\left.\quad \Delta f_{i}=0, \Delta \lambda_{i}=0, \Delta \bar{\beta}=0\right\}
\end{aligned}
$$

becomes an attractive manifold for (25). It implies the local asymptotic convergence of the tracking errors, i.e., $\Delta \Lambda \rightarrow 0 \Rightarrow\left(\Delta f_{i}, \Delta \lambda_{i}, \Delta \bar{\beta}\right) \rightarrow(0,0,0)$, as well as grasping with well-posed smooth control signals, without measurements of deformation, neither the contact area nor the contact force.

Proof. A passivity-based analysis of the closed loop system yields, for input $\boldsymbol{u}_{c l}$ and output $\boldsymbol{y}_{c l}=\boldsymbol{\nu}$,

$$
\begin{aligned}
\boldsymbol{u}_{c l}^{T} \boldsymbol{y}_{c l}= & \frac{\mathrm{d}}{\mathrm{d}} t \\
& +\dot{\boldsymbol{q}}_{1}^{T} \boldsymbol{K}_{q_{1}} \dot{\boldsymbol{q}}_{1}+\dot{\boldsymbol{q}}_{2}^{T} \boldsymbol{K}_{q_{2}} \dot{\boldsymbol{q}}_{2} \\
& +\sum_{i=1}^{2} \bar{r}_{i}\left[\dot{x}_{i}\left(\boldsymbol{q}_{i}\right) \quad \dot{y}_{i}\left(\boldsymbol{q}_{i}\right)\right]\left[\begin{array}{c}
s\left(\alpha_{i}\right) \\
c\left(\alpha_{i}\right)
\end{array}\right] F_{\beta} \\
& \left.-\left(\begin{array}{ll}
\dot{x} & \dot{y}
\end{array}\right]\left[\begin{array}{l}
\bar{r}_{1} s\left(\alpha_{1}\right)+\bar{r}_{2} s\left(\alpha_{2}\right) \\
\bar{r}_{1} c\left(\alpha_{1}\right)+\bar{r}_{2} c\left(\alpha_{2}\right)
\end{array}\right]\right) F_{\beta} \\
& +\dot{\theta} R f_{d} \frac{1}{2} D_{12} C S+\dot{\theta} R \frac{1}{2} M g D C_{12} \\
= & \frac{\mathrm{d}}{\mathrm{d} t} \bar{E}(\overline{\boldsymbol{\nu}}, \dot{\boldsymbol{\nu}})+\dot{\boldsymbol{q}}^{T} \boldsymbol{K}_{q} \dot{\boldsymbol{q}}+K_{\beta_{v}}\left(r_{1} \dot{\alpha}_{1}-r_{2} \dot{\alpha}_{2}\right)^{2} \\
& +\dot{\theta} R f_{d} \frac{1}{2} D_{12} C S+\dot{\theta} R \frac{1}{2} M g D C_{12},
\end{aligned}
$$

where $\boldsymbol{K}_{q}=\operatorname{diag}\left(\boldsymbol{K}_{q_{1}}, \boldsymbol{K}_{q_{2}}\right)$, and $\bar{E}(\overline{\boldsymbol{\nu}}, \dot{\boldsymbol{\nu}})=$ $K(\boldsymbol{q}, \dot{\boldsymbol{\nu}})+\Delta P_{E}(\overline{\boldsymbol{\nu}})+P_{\text {art }}(\overline{\boldsymbol{\nu}}) \in \mathbb{R}$ represents the closed-loop storage energy function of the error system, $\Delta P_{E}(\overline{\boldsymbol{\nu}}) \in \mathbb{R}$ models the elastic potential energy error of the system, $P_{\text {art }}(\overline{\boldsymbol{\nu}})=\frac{1}{2} K_{\beta_{p}}\left(r_{1} \alpha_{1}-r_{2} \alpha_{2}\right)^{2}$ is an artificial potential representing a virtual torsional spring to drive $\Delta \bar{\beta} \rightarrow 0$ that complies to the quality of grasp for $\beta=\pi$. To obtain (30), we have resorted to

$$
\begin{aligned}
r_{1} \dot{\alpha}_{1}-r_{2} \dot{\alpha}_{2}= & \bar{r}_{1}\left[\begin{array}{ll}
\dot{x}_{1}\left(\boldsymbol{q}_{1}\right) & \dot{y}_{1}\left(\boldsymbol{q}_{1}\right)
\end{array}\right]\left[\begin{array}{l}
s\left(\alpha_{1}\right) \\
c\left(\alpha_{1}\right)
\end{array}\right] \\
& +\bar{r}_{2}\left[\begin{array}{ll}
\dot{x}_{2}\left(\boldsymbol{q}_{2}\right) & \dot{y}_{2}\left(\boldsymbol{q}_{2}\right)
\end{array}\right]\left[\begin{array}{l}
s\left(\alpha_{2}\right) \\
c\left(\alpha_{2}\right)
\end{array}\right] \\
& -\left[\begin{array}{ll}
\dot{x} & \dot{y}
\end{array}\right]\left[\begin{array}{l}
\bar{r}_{1} s\left(\alpha_{1}\right)+\bar{r}_{2} s\left(\alpha_{2}\right) \\
\bar{r}_{1} c\left(\alpha_{1}\right)+\bar{r}_{2} c\left(\alpha_{2}\right)
\end{array}\right] \\
= & r_{1} \dot{\boldsymbol{q}}_{1}^{T} \boldsymbol{e}_{1}+r_{2} \dot{\boldsymbol{q}}_{2}^{T} \boldsymbol{e}_{2} .
\end{aligned}
$$

Now, since $r_{1}=r_{2}$, we have that

$$
\begin{aligned}
D_{12} & =\frac{R\left(r_{2}-r_{1}\right)+\delta_{2} r_{1}-\delta_{1} r_{2}}{r_{1} r_{2}} \leq 1, \\
D C_{12} & \leq \frac{R}{r_{1}}+2,
\end{aligned}
$$




$$
\begin{aligned}
C S & \leq 1 \\
\| \dot{\theta} R & \frac{1}{2}\left(f_{d} D_{12} C S+\frac{1}{2} M g D C_{12}\right) \| \\
& \leq \dot{\theta} R \frac{1}{2}\left(f_{d}+M g\right)\left(\frac{R}{r_{1}}+2\right) .
\end{aligned}
$$

By virtue of $\boldsymbol{u}_{c l}=0$, 30 becomes

$$
\dot{\bar{E}}(\overline{\boldsymbol{\nu}}, \dot{\boldsymbol{\nu}}) \leq-\dot{\boldsymbol{q}}^{T} \boldsymbol{K}_{q} \dot{\boldsymbol{q}}-K_{\beta_{v}}\left(r_{1} \dot{\alpha}_{1}-r_{2} \dot{\alpha}_{2}\right)^{2}-\dot{\theta} \gamma
$$

where

$$
\gamma=\frac{1}{2} R\left(f_{d}+M g\left(\frac{R}{r_{1}}+2\right)\right) \in \mathbb{R}^{+} .
$$

Since $\bar{E}(\overline{\boldsymbol{\nu}}, \dot{\boldsymbol{\nu}})$ is not a Lyapunov function in the primary constrained manifold, we cannot invoke Lyapunov theorems to show stability. However, this energy-like function can be used to analyze the stability of the closed-loop autonomous system on the largest invariant set. To this end, notice that (31) has two possible annihilators: (i) $(\dot{\boldsymbol{q}}, \dot{\theta})=(\mathbf{0}, 0)$ or (ii) $\dot{\theta}=$ $\gamma^{-1}\left(\sum_{i=1,2} \dot{\boldsymbol{q}}_{i}^{T} \boldsymbol{K}_{q_{i}} \dot{\boldsymbol{q}}_{i}+K_{\beta_{v}}\left(r_{1} \dot{\alpha}_{1}-r_{2} \dot{\alpha}_{2}\right)^{2}\right)$.

Moreover, the equilibrium (ii) implies a rather large angular velocity of the object, which stands for a rare and impractical operational regime that can be avoided locally by initial conditions and tuning; conversely, condition (i) can be obtained easily with joint damping large enough through theoretically large $\boldsymbol{K}_{q_{i}}$ for $|\dot{\theta}| \leq \epsilon_{0}$ and $|\gamma| \leq \epsilon_{1}$ with $\epsilon_{0}, \epsilon_{1}>0$, henceforth (31) is locally negative semi-definite. Consequently, in (i), 31) becomes negative semi-definite along the solution trajectories with the equilibrium at $(\dot{\boldsymbol{q}}, \dot{\theta})=(\mathbf{0}, 0)$, which implies that $\dot{\nu}=\mathbf{0}$. In this condition, 25 becomes

$$
\boldsymbol{A} \Delta \Lambda=\mathbf{0} .
$$

The coupling matrix $\boldsymbol{A}$ is non-degenerate if $\boldsymbol{J}_{i}\left(\boldsymbol{q}_{i}\right)$ is full column rank, which is fulfilled for $\boldsymbol{q}_{i 1} \neq a \pi$, with $a \in \mathbb{Z}$, for $i=1,2$. This configuration represents a rather awkward pose with the finger phalanges collapsing on themselves, which is avoided through proper initialization in any reasonable practical experiment; thus, excluding this configuration, we have that $\boldsymbol{A}$ is a locally non-degenerate matrix. Hence, the unique solution of (32) is $\Delta \Lambda=0$, which implies that locally asymptotically

$$
\begin{aligned}
\text { force errors: } & \Delta f_{i} \rightarrow 0, \Delta \lambda_{i} \rightarrow 0, \\
\text { optimal pose: } & F_{\beta} \rightarrow 0, \\
\text { aligment: } & D_{12} \rightarrow 0, D C_{12} \rightarrow 0
\end{aligned}
$$

without resorting to force feedback, neither deformation nor contact area measurement.
Remark 2. We have assumed that $r_{1}=r_{2}$, so with no loss of generality $\Delta \bar{\beta}=0$ means that $\alpha_{1}-$ $\alpha_{2}=0$. This chain of implications leads to the conclusion that the normal force vectors are dynamically aligned passing through the $C o M$ without involving any complex motion planning scheme. This stands for an optimal dynamic balanced grasp, in energetic terms, since normal and tangential forces are minimal. At this point, $\Delta C S_{i}=\Delta S C_{i}=0$ implies $f_{i}\left(\delta_{i}\right)=$ $F_{d_{i}}=f_{d}-(-1)^{i} M g \sin \left(\alpha_{i}\right) / 2$ and $\lambda_{i}=\lambda_{d_{i}}=$ $D_{i}\left(\boldsymbol{q}_{i}, \overline{\boldsymbol{p}}\right) M g \sin \left(\alpha_{i}\right) /\left(2 r_{i}\right)$; see (15) and (16). Finally, this represents the convergence of each finger to the best normal and tangential forces that allow compensating for gravity with an optimal grasping.

Remark 3. Theorem 1 establishes precise conditions for grasping with a given quality encoded in $\beta_{d}$, although at the expense of a slow convergence rate due to a high damping to meet the condition (i) discussed in the proof. Moreover, the slow dynamics of fingertip deformation imply that the injection of $\lambda_{i}$ into the underactuated object dynamics is also slow. Overall, owing to a slow convergence rate toward the basic manifold, it is expected, intuitively and theoretically, as if this were mimicking a robot learning from scratch to pinch optimally an object, which certainly constitutes a high-end task.

\section{Simulations and experiments}

4.1. Simulator. The simulator is programmed in Matlab 7.13, based on a variation of the Bogacki-Shampine variable-step stiff numerical integrator (Bogacki and Shampine, 1989), implemented as ode23tb with a maximum step size of $4 \mathrm{~ms}$ and an absolute error tolerance of $1 \times 10^{-6} \mathrm{~m}$. A constrained stabilization method (CSM) (Baumgarte, 1971) is introduced for numerical stabilization of the DAE-2 system, whose overall initial conditions are consistent with the DAE formulation of the system for $t=t_{0}$ such that $f_{i}(0)>0$, for $i=1,2$, and the initial grasp is validated.

\subsection{Simulation conditions.}

4.2.1. System parameters. Physical parameters of two equal robotic fingers of $3 \mathrm{DoFs}$ each are described in Table 1, where $i, j$ represent the link and the robot finger, respectively. Thus $L_{j i}, L_{c m_{j i}}, M_{j i}$ and $I_{j i}$ stand for the link length, distance to the center of mass from the $i$-th joint, mass, and mass moment of inertia, respectively. Additionally, $M=0.20121 \mathrm{~kg}, I=8.050494 \times 10^{-4}$ $\mathrm{kgm}^{2}$ and $R=0.08 \mathrm{~m}$ is the mass, the mass moment of inertia and the radius of the object, respectively. Finally the $i$-th hemispherical soft-fingertip has a stiffness parameter of $k_{i}=10000 \pi \mathrm{kgm} / \mathrm{s}^{2} \mathrm{~m}^{2}$, with radius $r_{i}=$ 


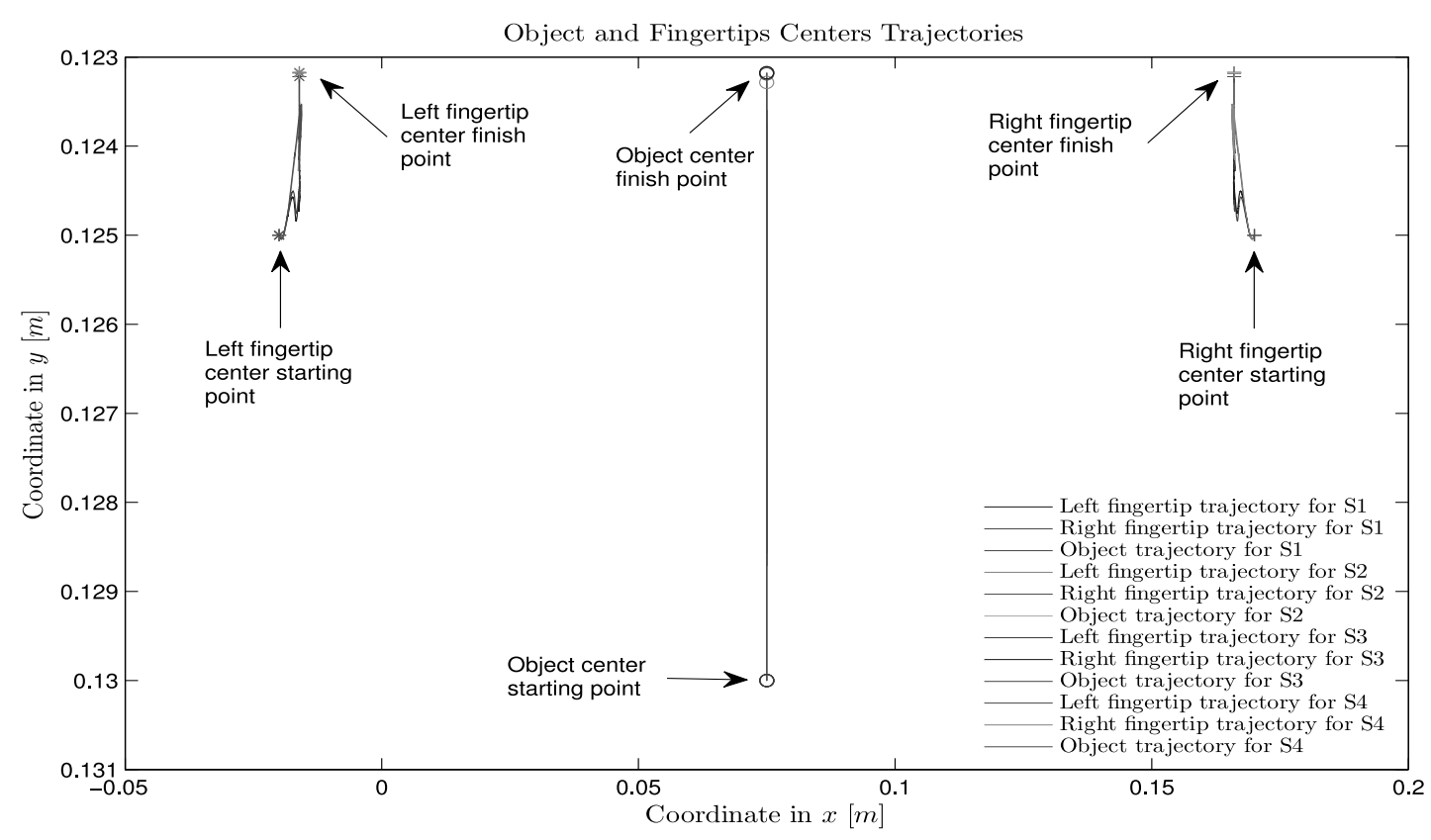

Fig. 3. Case 1: trajectories of fingertips and object centers in the system task space; as a quasi-horizontal line is conformed by normal forces, the optimal grasping condition $\beta(\overline{\boldsymbol{\nu}})=\pi \mathrm{rad}$ is achieved.

Table 1. Physical parameters of the robotic fingers.

\begin{tabular}{|c|c||c|c|}
\hline$L_{i 1}$ & $0.120 \mathrm{~m}$ & $M_{i 1}$ & $0.50062 \mathrm{~kg}$ \\
\hline$L_{i 2}$ & $0.08485 \mathrm{~m}$ & $M_{i 2}$ & $0.34045 \mathrm{~kg}$ \\
\hline$L_{i 3}$ & $0.040 \mathrm{~m}$ & $M_{i 3}$ & $0.1693 \mathrm{~kg}$ \\
\hline$L_{c m_{i 1}}$ & $0.05791 \mathrm{~m}$ & $I_{i 1}$ & $6.55341 \times 10^{-4} \mathrm{kgm}^{2}$ \\
\hline$L_{c m_{i 2}}$ & $0.03988 \mathrm{~m}$ & $I_{i 2}$ & $2.52286 \times 10^{-4} \mathrm{kgm}^{2}$ \\
\hline$L_{c m_{i 3}}$ & $0.0157 \mathrm{~m}$ & $I_{i 3}$ & $5.39262 \times 10^{-5} \mathrm{kgm}^{2}$ \\
\hline
\end{tabular}

Table 2. Control laws gains and desired normal force.

\begin{tabular}{|c|c|c|c|}
\hline $\begin{array}{c}\text { Sim } \\
\text { ID }\end{array}$ & $\begin{array}{c}\boldsymbol{K}_{q_{i}} \text { gain } \\
{\left[\mathrm{kgm}^{2} / \mathrm{s}\right]}\end{array}$ & $\begin{array}{c}K_{\beta_{p}} \text { gain } \\
{\left[\mathrm{kgm} / \mathrm{s}^{2}\right]}\end{array}$ & $\begin{array}{c}K_{\beta_{v}} \text { gain } \\
{[\mathrm{kg} / \mathrm{s}]}\end{array}$ \\
\hline \hline S1 & $\operatorname{diag}(0.5)$ & 300 & 250 \\
\hline S2 & $\operatorname{diag}(0.5)$ & 300 & 0 \\
\hline S3 & $\operatorname{diag}(0.5)$ & 600 & 250 \\
\hline S4 & $\operatorname{diag}(0.5)$ & 600 & 0 \\
\hline
\end{tabular}

$0.02 \mathrm{~m}$ such that the object is four times bigger than the fingertip.

4.2.2. Simulation conditions. For comparative purposes, two simulation cases are considered under different initial conditions and sets of feedback gains with $f_{d}=2.5\left[\mathrm{kgm} / \mathrm{s}^{2}\right]$, as shown in Table 2. The initial conditions should guarantee that $\delta_{i}(0)>0$, such that $f_{i}(0)>0$. The integration interval for all simulations is from 0 to $10 \mathrm{~s}$, and some graphs have been cropped to show some particular details.

Case 1. The system is initialized with $\alpha_{1}(0)=-\alpha_{2}(0)=$ $-0.21250 \mathrm{rad}$, which means an internal angle of $\bar{\beta}(0)=$ 2.71658 rad. The initial conditions are $\boldsymbol{q}_{1}(0)=\boldsymbol{q}_{2}(0)=$ $[0.42685,1.77089,1.17068]^{T} \mathrm{rad}$ and $\dot{\boldsymbol{q}}_{1}(0)=\dot{\boldsymbol{q}}_{2}(0)=$ $[0,0,0]^{T} \mathrm{rad} / \mathrm{s}$ for fingers, and $\overline{\boldsymbol{p}}(0)=[0.075,0.130]^{T} \mathrm{~m}$, $\theta(0)=0 \mathrm{rad}$ and $\dot{\overline{\boldsymbol{p}}}(0)=[0,0]^{T} \mathrm{~m} / \mathrm{s}, \dot{\theta}(0)=0 \mathrm{rad} / \mathrm{s}$ for the object, and with $\delta_{1}(0)=\delta_{2}(0)=0.00232 \mathrm{~m}$ such that $f_{1}(0)=f_{2}(0)=0.17011 \mathrm{kgm} / \mathrm{s}^{2}$.
Case 2. The system is initialized with $\alpha_{1}(0)=-0.21250$ rad and $\alpha_{2}(0)=0.05258 \mathrm{rad}$, which means an internal angle of $\bar{\beta}(0)=2.87650 \mathrm{rad}$. The initial conditions are $\boldsymbol{q}_{1}(0)=[0.42685,1.77089,1.17068]^{T} \mathrm{rad}, \boldsymbol{q}_{2}(0)=$ $[0.42685,1.77089,0.77798]^{T} \operatorname{rad}$ and $\dot{\boldsymbol{q}}_{1}(0)=\dot{\boldsymbol{q}}_{2}(0)=$ $[0,0,0]^{T} \mathrm{rad} / \mathrm{s}$ for fingers, and $\overline{\boldsymbol{p}}(0)=[0.075,0.130]^{T}$ $\mathrm{m}, \theta(0)=0 \mathrm{rad}$ and $\dot{\overline{\boldsymbol{p}}}(0)=[0,0]^{T} \mathrm{~m} / \mathrm{s}, \dot{\theta}(0)=0$ $\mathrm{rad} / \mathrm{s}$ for the object, and with $\delta_{1}(0)=0.00232 \mathrm{~m}$ and $\delta_{2}(0)=0.00486 \mathrm{~m}$ such that $f_{1}(0)=0.17011 \mathrm{kgm} / \mathrm{s}^{2}$ and $f_{2}(0)=0.74463 \mathrm{kgm} / \mathrm{s}^{2}$.

\subsection{Simulation results.}

4.3.1. Results of Case 1. Figure 3 shows the system movement to achieve stable grasping conditions, that is, the alignment of the centers of fingertips and the object in a quasi-horizontal line, while Fig. 4 shows how the object is lifted in order to achieve the stable grasping conditions. The error convergence of normal and 
tangential forces is shown in Figs. 5] and 6, respectively, where it can be appreciated that the effect due to gravity is compensated accordingly. On the other hand, Fig. 7 shows the convergence of internal angles $\alpha_{1}\left(\boldsymbol{q}_{1}, \overline{\boldsymbol{p}}\right)$ and $\alpha_{2}\left(\boldsymbol{q}_{2}, \overline{\boldsymbol{p}}\right)$, which implies that both angles converge to the same value, that is, the convergence to the optimal stable grasping angle $\bar{\beta}=\pi$; see Fig. 8. Finally, if we analyse the differences between simulations (for the four different sets of feedback gains), we can see a direct correlation of gain $K_{\beta_{p}}$ with the velocity of the system to reach stable grasping conditions with some oscillations. On the other hand, the gains $\boldsymbol{K}_{q_{i}}$ are directly correlated to the damping of fingers, which is used in order to achieve asymptotic convergence for the grasping conditions; furthermore, the gain $K_{\beta_{v}}$ is also related to damping, but as such damping is dependent on angles $\dot{\alpha}_{1}\left(\boldsymbol{q}_{1}, \overline{\boldsymbol{p}}\right)$ and $\dot{\alpha}_{2}\left(\boldsymbol{q}_{2}, \overline{\boldsymbol{p}}\right)$, it is more suitable for reducing the oscillatory behaviour of internal angles as the stable grasping conditions are achieved. Therefore, a correct balance between this pair of control gains must be established in order to achieve a desired system performance.

4.3.2. Results of Case 2. Figure 9 shows the system movement to achieve stable grasping conditions, that is, the alignment of the centers of the fingertips and the object in a line, which is diagonal due the initial conditions of the system, while Fig. 10 shows how the object is lifted but also rotated, as the left finger displaces and rolls a greater distance achieving the grasping. The error convergence of normal and tangential forces is shown in Figs. 11 and 12 , respectively, where we can highlight that the gravitational effect is compensated. On the other hand, in Fig. 13 the convergence of internal angles $\alpha_{1}\left(\boldsymbol{q}_{1}, \overline{\boldsymbol{p}}\right)$ and $\alpha_{2}\left(\boldsymbol{q}_{2}, \overline{\boldsymbol{p}}\right)$, is shown and it is possible to corroborate how both angles converge to the same value but not necessarily to zero, and where $\alpha$ angles may vary once they reach the same
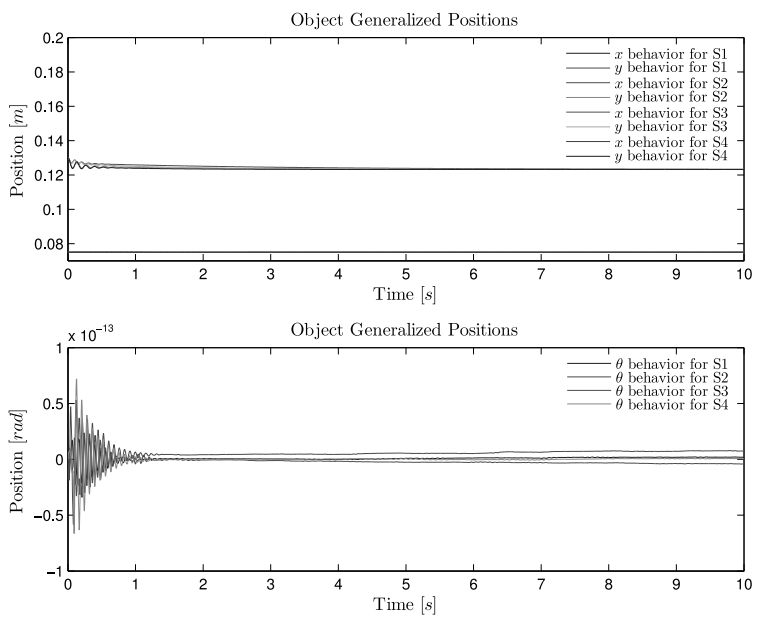

Fig. 4. Case 1: smooth behavior of object coordinates/positions.
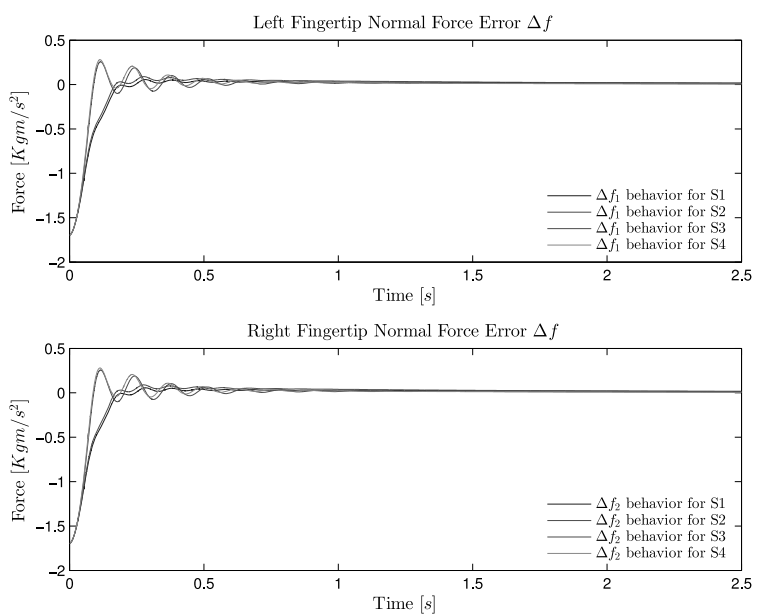

Fig. 5. Case 1: after a short smooth transient, normal forces errors converge to zero, showing a dynamic search of the optimal configuration.

value if any remaining rotational movement of the object exists. If the object rotation is not stopped due the friction induced by the control laws, eventually the stable grasp can be lost, since it was discussed as an ideal stable grasp condition, where control gains $\boldsymbol{K}_{q_{i}}$ and $K_{\beta_{v}}$ must be large enough but $K_{\beta_{p}}$ small. Finally, from the simulations we can conclude similar conclusions in the relationships between control gains and the performance of the system as for the previous case.

\subsection{Experimental results.}

4.4.1. Experimental setup. In order to verify the performance of the proposed controller in a real setting, a pair of robotic fingers with soft-tips and three degrees each
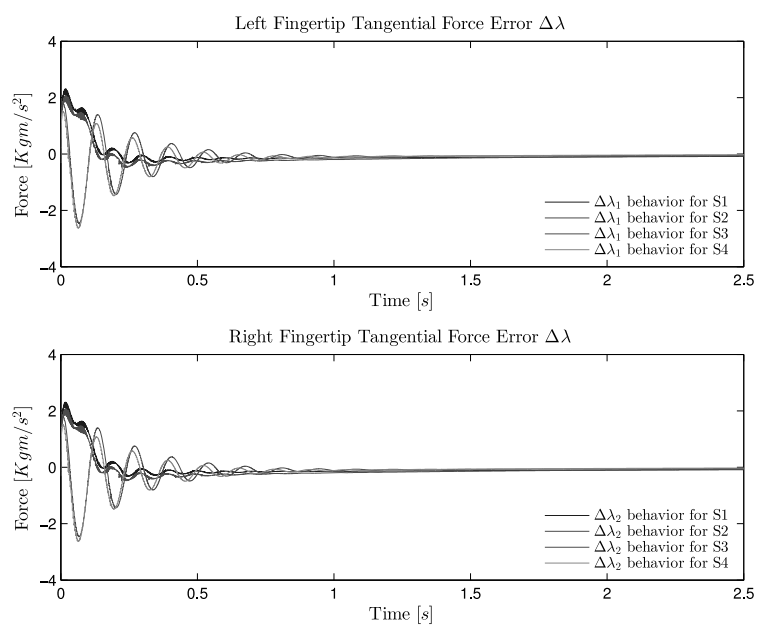

Fig. 6. Case 1: after a short smooth transient, tangential forces errors converge to zero, compensating the object weight. 


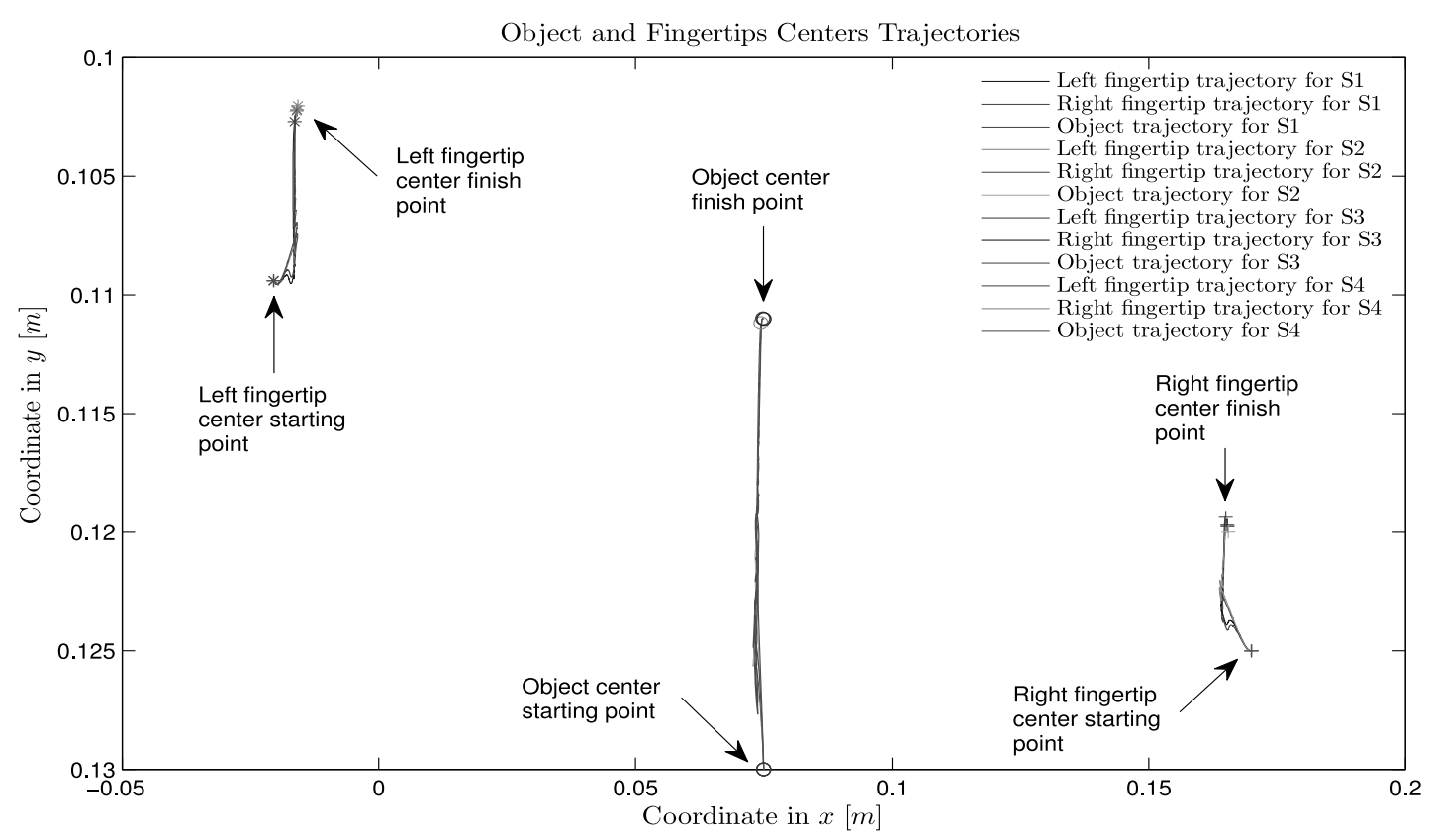

Fig. 9. Case 2: trajectories of fingertips and object centers in the system task space; as a diagonal line is conformed by normal forces, the optimal grasping condition $\beta(\overline{\boldsymbol{\nu}})=\pi \mathrm{rad}$ is achieved.

was implemented as a testbed; see Fig. 15. In each finger we used Dynamixel ${ }^{\circledR}$ MX-64R servos in torque mode. Generalized positions and velocities of the fingers are obtained directly from the servomotors while the position of the object's center of mass is obtained through a web camera, QuickCam ${ }^{\circledR}$ E500 ${ }^{\mathrm{TM}}$ from Logitech, using the OpenCV library. Finally, the experiments were developed using $\mathrm{C}++$ on a PC under Ubuntu 14.04 LTS equipped with an RTAI patch; see Fig. 16

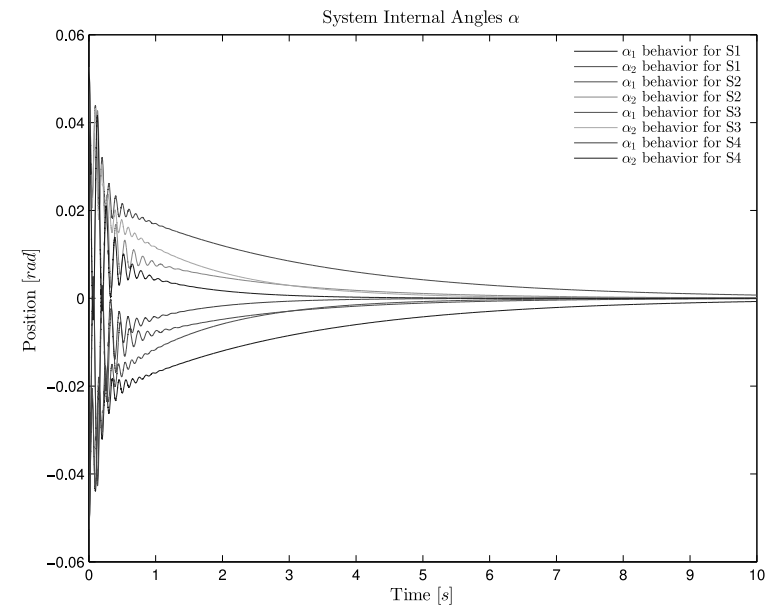

Fig. 7. Case 1: smooth convergence of both $\alpha$ angles to a common value close to zero.
4.5. Experimental parameters and conditions. The physical parameters of the robotic fingers are $L_{i 1}=0.088$ $\mathrm{m}, L_{i 2}=0.088 \mathrm{~m}, L_{i 3}=0.036 \mathrm{~m}, M_{i 1}=0.2, M_{i 2}=$ $0.2 \mathrm{~kg}, M_{i 3}=0.15 \mathrm{~kg}, L_{c m_{i 1}}=0.06 \mathrm{~m}, L_{c m_{i 2}}=0.06 \mathrm{~m}$ and $L_{c m_{i 3}}=0.025 \mathrm{~m}$ for indices $i, j$ representing the link and the robot finger, respectively. Additionally, $M=0.04$ $\mathrm{kg}$ and $R=0.05 \mathrm{~m}$ are the mass and radius of the object, respectively. Finally, the $i$-th hemispherical soft-fingertip

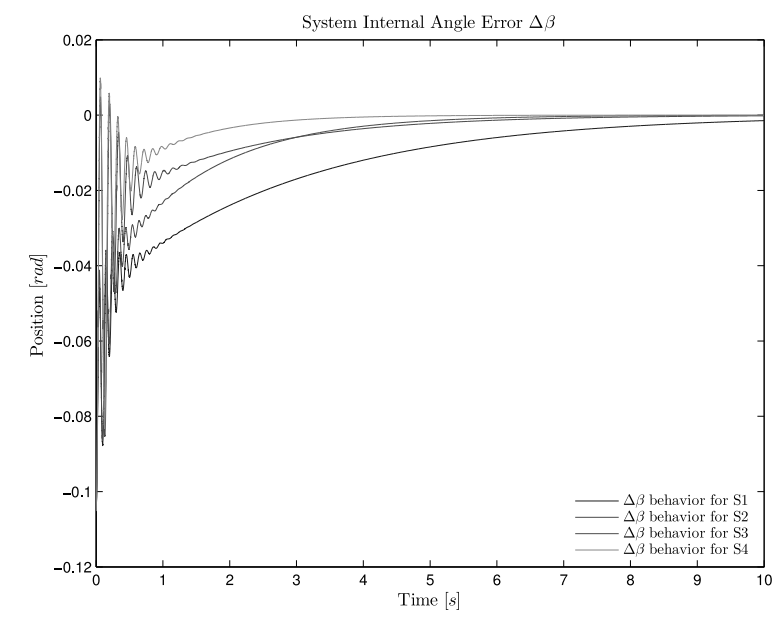

Fig. 8. Case 1: after a short smooth transient, exponential-like convergence of the optimal grasping error $\Delta \beta(\overline{\boldsymbol{\nu}})$ is achieved. 
has a stiffness 2 parameter of $k_{i}=270563.4 \mathrm{kgm} / \mathrm{s}^{2} \mathrm{~m}^{2}$, with radius $r_{i}=0.03 \mathrm{~m}$, and $L_{0}=0.20 \mathrm{~m}$ is the distance between each finger's base.

As in the previous section, we consider $f_{d}=2.5$ $\left[\mathrm{kgm} / \mathrm{s}^{2}\right]$ while the system is initialized with $\alpha_{1}(0)=$ $-0.027 \mathrm{rad}$ and $\alpha_{2}(0)=-0.17 \mathrm{rad}$. The initial conditions of the fingers are $\boldsymbol{q}_{1}(0)=[1.20111,0.670349,1.39592]^{T}$ $\mathrm{rad}, \boldsymbol{q}_{2}(0)=[0.228563,1.46955,0.0901538]^{T} \mathrm{rad}$ and $\dot{\boldsymbol{q}}_{1}(0)=\dot{\boldsymbol{q}}_{2}(0)=[0,0,0]^{T} \mathrm{rad} / \mathrm{s}$ for fingers, and $\overline{\boldsymbol{p}}(0)=$ $[0.160923,0]^{T} \mathrm{~m}, \theta(0)=0 \mathrm{rad}$ and $\dot{\overline{\boldsymbol{p}}}(0)=[0,0]^{T}$ $\mathrm{m} / \mathrm{s}, \dot{\theta}(0)=0 \mathrm{rad} / \mathrm{s}$ for the object, $K_{q i}=0.1 \mathrm{kgm}^{2} / \mathrm{s}$, $K_{\beta p}=125 \mathrm{kgm} / \mathrm{s}^{2}, K_{\beta v}=10 \mathrm{~kg} / \mathrm{s}$.

The objective is to guarantee stable grasping of

\footnotetext{
${ }^{2}$ The deformable tips are made with resin, RESILAM 95/M, while the stiffness parameter was obtained experimentally.
}
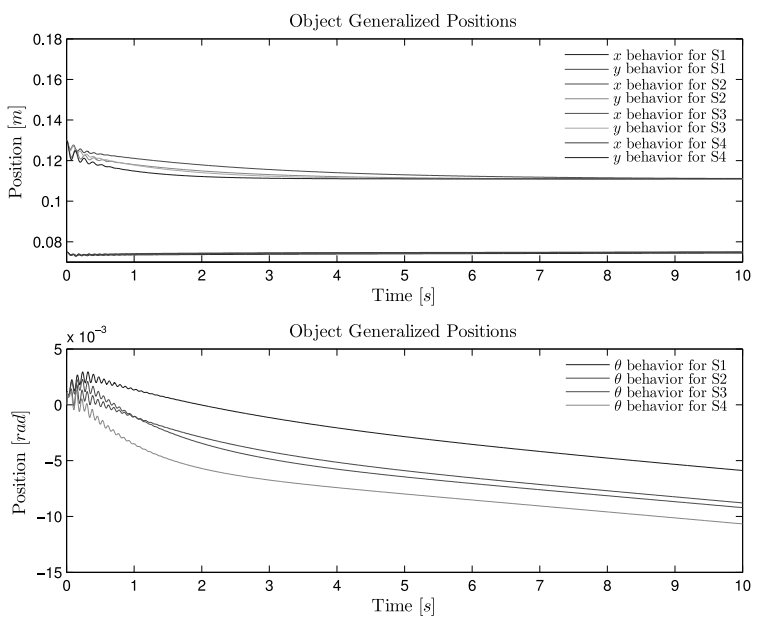

Fig. 10. Case 2: smooth behavior of object coordinates/positions.
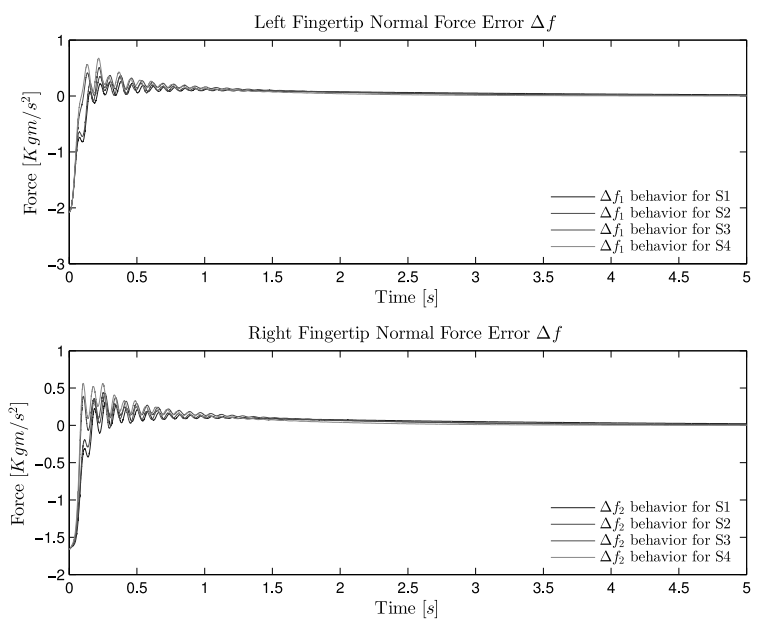

Fig. 11. Case 2: after a short smooth transient, normal forces errors converge to zero, showing a dynamic search of the optimal configuration. a circular object based on the optimal stable grasping. In Fig. 17 we can notice the contribution of the hemispherical and deformable tips of the fingers to align the centers of fingertips and the object, while in Fig. 18the convergence of $\alpha_{1}\left(\boldsymbol{q}_{1}, \overline{\boldsymbol{p}}\right)$ and $\alpha_{2}\left(\boldsymbol{q}_{2}, \overline{\boldsymbol{p}}\right)$ approximately to the same value but not necessarily to zero is shown. The variations around 0.1 represent small errors in the detection of the object; these variations occur due to light changes which the camera translates as movements of the object (these can be perfectly seen in Fig. 17 when the optimal grasping is reached). Finally, in Figs. 19 and 20 the exponential convergence of the angle error for stable grasping in approximately $1 \mathrm{~s}$ and the force errors, respectively, is shown.
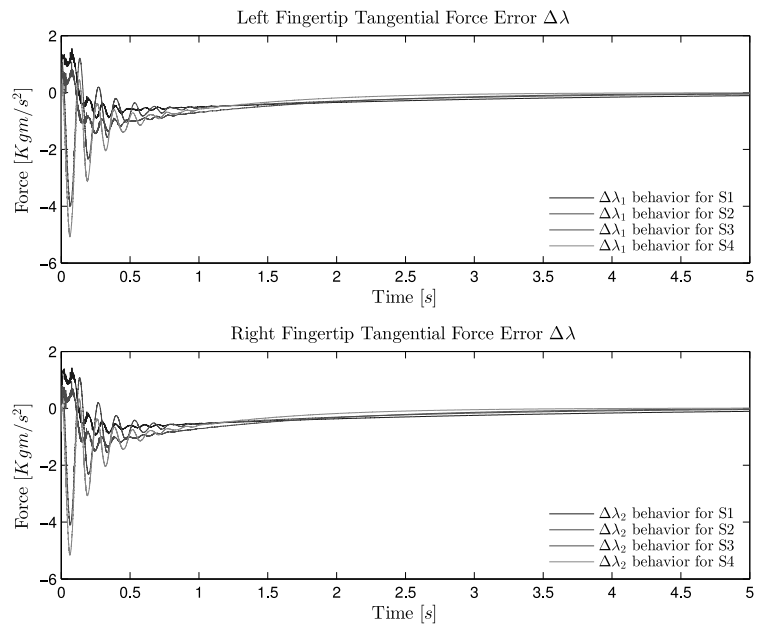

Fig. 12. Case 2: after a short smooth transient, tangential forces errors converge to zero, compensating the object weight.

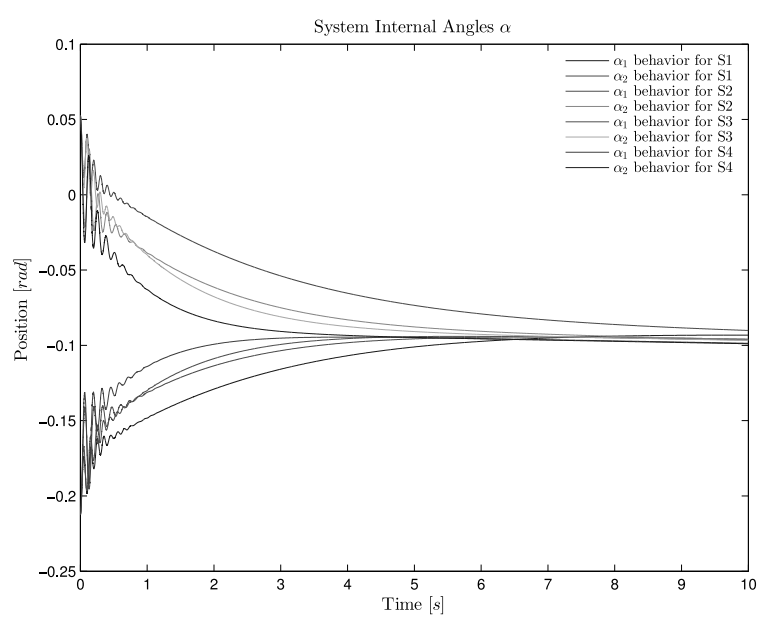

Fig. 13. Case 2: smooth convergence of both $\alpha$ angles to a common value not necessarily at zero, which can be changing until the remaining object rotation is stopped. 


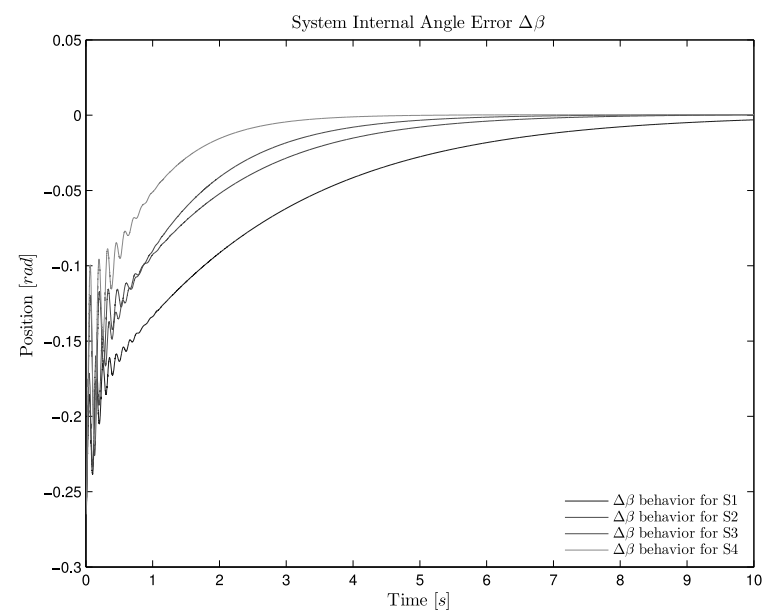

Fig. 14. Case 2: after a short smooth transient, exponential-like convergence of the optimal grasping error $\Delta \beta(\overline{\boldsymbol{\nu}})$ is achieved.

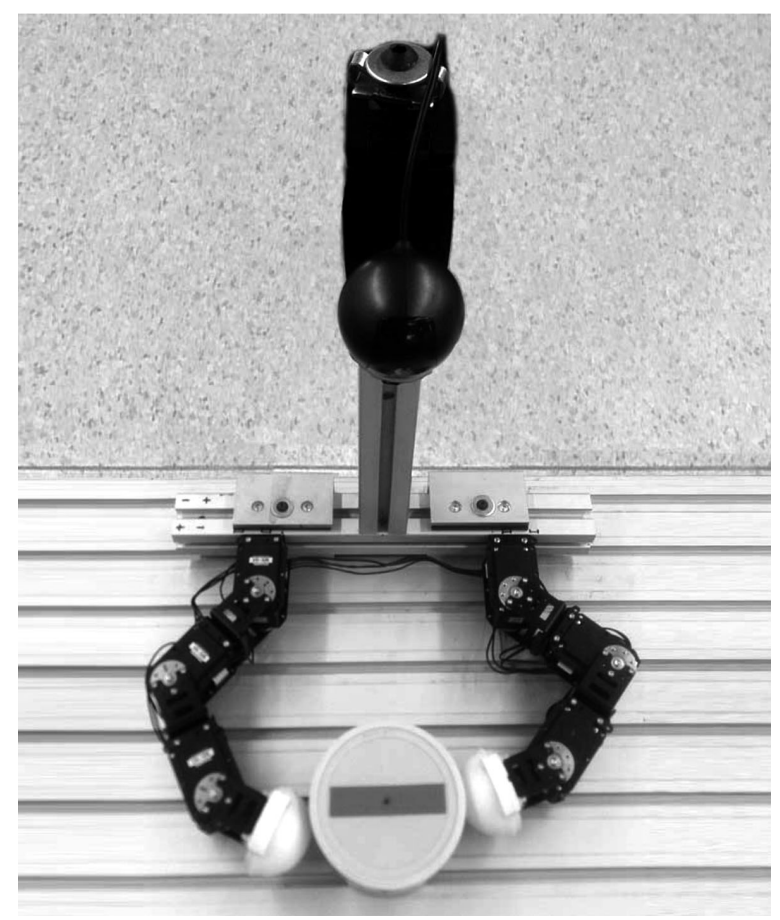

Fig. 15. Experimental setup.

\section{Final remarks}

The quality of grasping rigid curved objects was addressed in this paper, using redundant robotic fingers with deformable fingertips. Despite the apparent complexity of the model and stability analysis, the controller is quite simple for the optimal grasping of a dynamic object subject to gravity where the normal forces are aligned, passing through the $C o M$ of the object. The final condition of the normal forces represents a trivial pose which

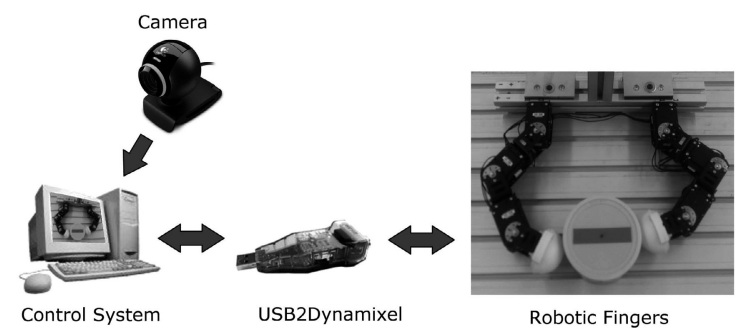

Fig. 16. General structure of the robotic fingers controller.

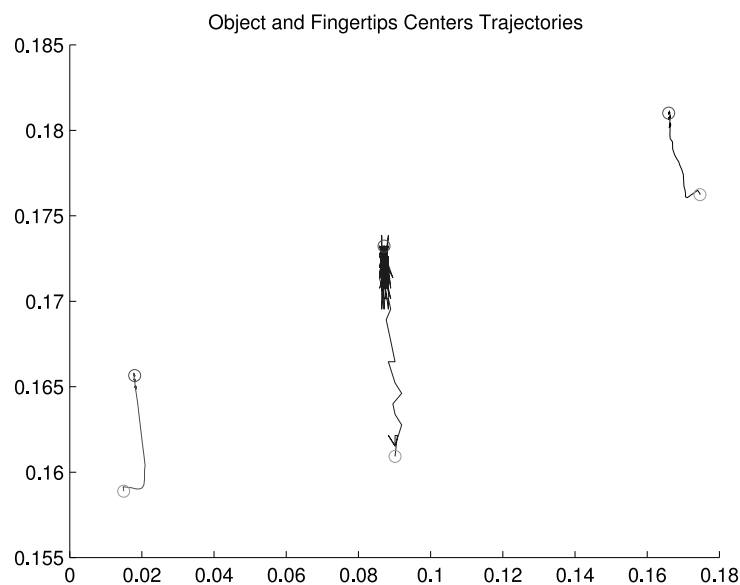

Fig. 17. Performance of the trajectories of fingertips and object centers in the system task space to achieve the optimal grasping condition $\beta(\bar{\nu})=\pi \mathrm{rad}$.

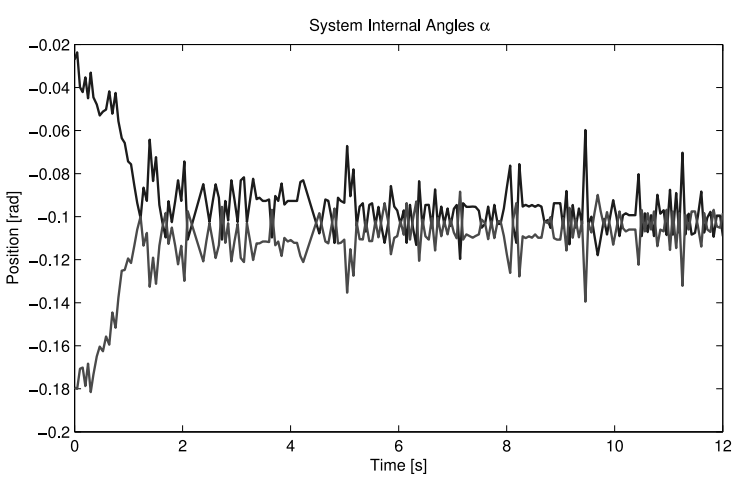

Fig. 18. Experimental performance of the angles $\alpha_{i}$.

requires a minimum energy, and this is possible by the rolling of the hemispherical fingertips into the circular object-a property that has not been explored previously in the context of an optimal grasping with the automatic 


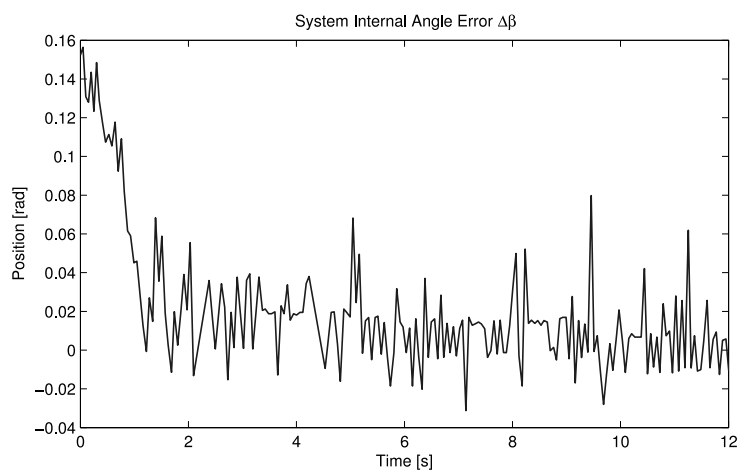

Fig. 19. Experimental performance of the optimal grasping error $\Delta \beta(\bar{\nu})$
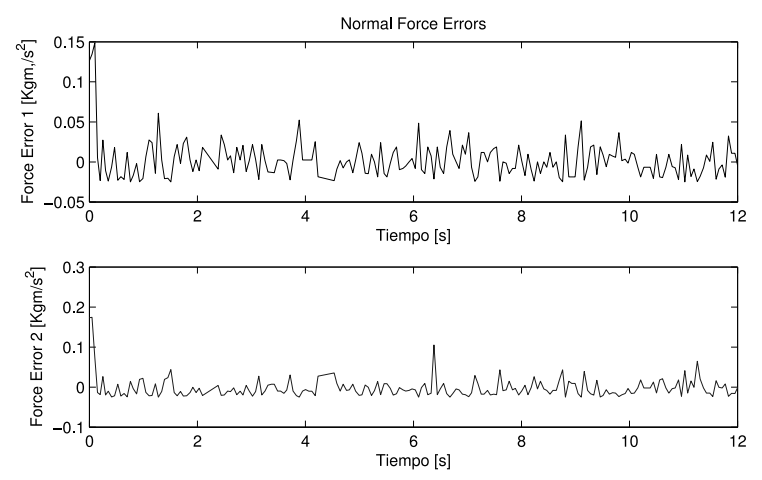

Fig. 20. Experimental performance of the force errors.

synthesis of the desired equilibrium. Notice that this approach exhibits an intuitive biomimetic performance to grasp an object, which is unknown or not evident for the grasping and manipulation based on the point contact.

Thus, an explicit inclusion of rolling in fingers with soft tips, characteristic that is fundamental in our human hands for precise manipulation, arises as a feasible option for dexterous tasks with robotic hands.

All these issues were verified in a real experimental setting throughout experiments. This approach can be extended to an object with arbitrary shape that admits a local decomposition by a circular curvature assuming that the objects can be defined as a set $X \in \mathbb{R}^{2}$ whose boundary $\partial X$ is a finite union of mutually disjoint simple closed curves. If an affine joint or contact friction is considered, it will only increase dissipativeness; our result applies in the presence of such friction. Extension to the tracking case, and thus manipulation, seems feasible in the realm of passivity; however, partial knowledge of the dynamics may be needed to compensate for kinetic energy in the error frame. The $3 \mathrm{D}$ case becomes more involved but straightforward for the regulation case, where the convex hull of the quality of grasp provides a conservative measure. Finally, the inclusion of the visual sensors under kinematic and dynamics uncertainties (Skrzypczyński, 2005) represents a more comprehensive system.

\section{Acknowledgment}

This work was partially supported by CONACYT-Mexico under the grants 133646 and 174597 as well the M.Sc. scholarship 263479, and by the Programme of International Scientific Cooperation CONICYT/CONACYT, grant 2011-380.

\section{References}

Akella, P. and Cutkosky, M. (1989). Manipulating with soft-fingers, IEEE International Conference on Robotics and Automation, Scottsdale, AZ, USA, pp. 767-769.

Arimoto, S. (2007). Control Theory of Multi Fingered Hands, Springer-Verlag, London.

Arimoto, S., Nguyen, P.T.A., Han, H.Y. and Doulgeri, Z. (2000). Dynamics and control of a set of dual fingers with soft tips, Robotica 18(1): 71-80.

Baumgarte, J. (1971). Stabilization of constraints and integrals of motion in dynamical systems, Computer Methods in Applied Mechanics and Engineering 1: 1-16.

Bogacki, P. and Shampine, L.F. (1989). A 3(2) pair of Runge-Kutta formulas, Applied Mathematics Letters 2(4): 321-325.

Coelho, J.A. and Grupen, R. (1994). Optimal multifingered grasp synthesis, IEEE International Conference on Robotics and Biomimetics, San Diego, CA, USA, pp. 1937-1942.

Cole, A., Hauser, J. and Sastry, S. (1989). Kinematics and control of multifingered hands with rolling contact, IEEE Transactions on Automatic Control 34(4): 398-404.

Harada, K. and Kaneko, M. (2001). Rolling based manipulation under neighborhood equilibrium, IEEE International Conference on Robotics and Automation, Seoul, Korea, pp. 2492-2498.

Ito, S., Mizukoshi, Y. and Sasaki, M. (2007). Numerical analysis for optimal posture of circular object grasped with frictions, IEEE/RSJ International Conference on Intelligent Robots and Systems, San Diego, CA, USA, pp. 2492-2498.

Jia, Y.B. (2000). Grasping curved objects through rolling, IEEE International Conference on Robotics and Automation, San Francisco, CA, USA, pp. 377-382.

Kim, B., Oh, S., Yi, B. and Suh, I.H. (2001). Optimal grasping based on non-dimensionalized performance indices, IEEE/RSJ International Conference on Intelligent Robots and Systems, Maui, HI, USA, pp. 949-956.

Marigo, A. and Bichi, A. (2007). Rolling bodies with regular surface: Controllability theory and applications, IEEE Transactions on Automatic Control 45(9): 1586-1599. 
Nakashima, A., Nagase, K. and Hayakawa, Y. (2005). Simultaneous control of grasping/manipulation and contact points with rolling contact, 16th IFAC World Congress, Prague, Czech Republic, pp. 415-420.

Nguyen, P.T.A., Ozawa, R. and Arimoto, S. (2006). Manipulation of a circular object by a pair of multi-DOF robotic fingers, IEEE/RSJ International Conference on Intelligent Robots and Systems, Beijing, China, pp. 5669-5674.

Ozawa, R., Arimoto, S., Nguyen, P.T.A., Yoshida, M. and Bae, J.H. (2004). Manipulation of a circular object in a horizontal plane by two finger robots, IEEE International Conference on Robotics and Biomimetics, Shenyang, China, pp. 517-522.

Ozawa, R., Arimoto, S., Nguyen, P.T.A., Yoshida, M. and Bae, J.H. (2005). Manipulation of a circular object without object information, IEEE/RSJ International Conference on Intelligent Robots and Systems, Edmonton, Alberta, Canada, pp. 1832-1838.

Parra-Vega, V., Rodriguez-Angeles, A., Arimoto, S. and Hirzinger, G. (2001). High precision constrained grasping with cooperative adaptive handcontrol, Journal of Intelligent and Robotic Systems 32(3): 235-254.

Salisbury, J. (1982). Kinematics and Force Analysis of Articulated Hands, Ph.D. thesis, Stanford University, Stanford, CA.

Shapiro, A. (2001). Force closure set of linearly controlled grasps, Technical report, Technion Israel Institute of Technology, Haifa.

Skrzypczyński, P. (2005). Uncertainty models of vision sensors in mobile robot positioning, International Journal of Applied Mathematics and Computer Science 15(1): 73-88.

Song, S., Park, J. and Choi, Y. (2012). Dual-fingered stable grasping control for an optimal force angle, IEEE Transactions on Robotics 28(1): 256-262.

Stramigioli, S. (2003). Modeling and IPC Control of Interactive Mechanical Systems-A Coordinate-free Approach, Lecture Notes in Control and Information Sciences, Vol. 266, Springer-Verlag, London.

Wen, S. and Wu, T. (2012). Computation for maximum stable grasping in dynamic force distribution, Journal of Intelligent Robot Systems 68: 225-243.

Wimboeck, T., Ott, C. and Hirzinger, G. (2006). Passivity-based object-level impedance control for a multifingered hand, IEEE/RSJ International Conference on Intelligent Robots and Systems, Beijing, China, pp. 4621-4627.
Yoshida, M., Arimoto, S. and Bae, J.-H. (2007). Blind grasp and manipulation of a rigid object by a pair of robot fingers with soft tips, IEEE International Conference on Robotics and Automation, Roma, Italy, pp. 4707-4714.

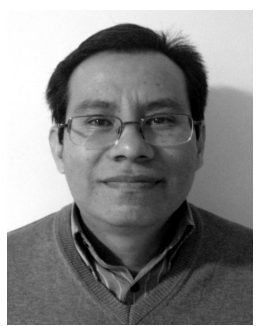

Rodolfo García-Rodríguez received a B.Sc. degree in industrial electronic engineering from Instituto Tecnológico de Puebla, México, in 1997. He received the Ph.D. and M.Sc. degrees from the Research Center for Advanced Studies of the National Polytechnic Institute (CINVESTAVIPN) in 2005 and 2002, respectively. In 19982000 he was with Gates Rubber de México in the Engineering Department. Currently, he is a professor at Universidad de los Andes, Chile. His research interests include robot control, robotic hands and bio-inspired control systems.

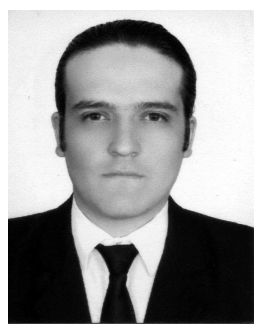

Victor Segogia-Palacios received the B.Eng. degree in mechatronics from the Polytechnic University of Pachuca, Hidalgo, México, in 2011. He received his M.Sc. degree in robotics and advanced manufacturing from the Center for Research and Advanced Studies of the $\mathrm{Na}$ tional Polytechnic Institute (CINVESTAV-IPN), Saltillo, México, in 2014. He has recently joined the private sector and has been working with firms related to the field of computer science and systems engineering in several projects. His research interest includes inertial and non-inertial system modeling, actuated and underactuated system control, non-linear stability analysis theory, force fontrol and robot cooperation, robot manipulation and unmanned aerial vehicle (UAV) robotics.

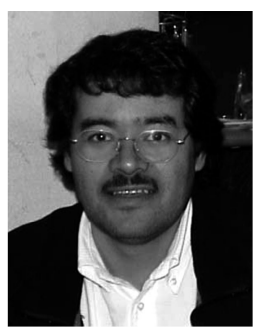

Vicente Parra-Vega received one B.Eng. degree in control and computing and another in electronics and communications, both from Nuevo León University, México, in 1987, the M.Sc. degree in automatic control from the Research Center for Advanced Studies of the National Polytechnic Institute (CINVESTAV-IPN), San Pedro Zacatenco, México, in 1989, and the Ph.D. degree in electrical engineering from the Mathematical Engineering and Information Physics Department of the University of Tokyo, Japan, in 1995, under the supervision of Prof. S. Arimoto. Currently, he is a full professor at the CINVESTAV. His present research interests include collaborative multirobots, high-precision servosystems, haptic interfaces, control theory, visual servoing, teleoperators, high-speed CNC, and unmanned aerial and submarine robots. He is the leading cofounder of two new postgraduate programmes in Mexico, one in mechatronics and the other in robotics and advanced manufacturing. Prof. Parra-Vega is a regular member of the Mexican Academy of Sciences, the National Researcher System, and since 1997 has been serving for several committees of the Mexican Council for Science and Technology. 


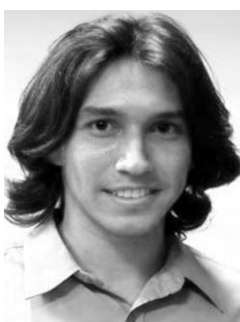

Marco Villalba-Lucio received his B.Eng. degree in mechatronics in 2013 from the Polytechnic University of Victoria, Mexico, and the M.Sc. degree in robotics and advanced manufacturing in 2015 from the Research Center for Advanced Studies (CINVESTAV), Saltillo, Mexico. His research interests include robot control, control of multi-fingered hands, fuzzy control and mechatronics systems.

Received: 17 April 2015

Revised: 23 October 2015

Re-revised: 28 December 2015

Accepted: 26 January 2016 